\title{
STATISTICAL RIEMANN PROBLEMS AND A COMPOSITION LAW FOR ERRORS IN NUMERICAL SOLUTIONS OF SHOCK PHYSICS PROBLEMS*
}

\author{
JAMES GLIMM ${ }^{\dagger}$,JOHN W. GROVE ${ }^{\ddagger}$, YONGHEE KANG ${ }^{\ddagger}$, TAEWON LEE ${ }^{\S}$, \\ XIAOLIN LI ${ }^{\S}$, DAVID H. SHARP ${ }^{\ddagger}$, YAN YU ${ }^{\S}$, KENNY YE ${ }^{\S}$, AND MING ZHAO
}

Abstract. We seek error models for shock physics simulations that are robust and understandable. The purpose of this paper is to formulate and validate a composition law to estimate errors in the solutions of composite problems in terms of the errors from simpler ones. We illustrate this idea in a simple context. This paper employs several simplifying assumptions (restriction to one spatial dimension, use of a simplified (gamma law) equation of state, and consideration of a single numerical method). In separate papers we will address the effect of these assumptions.

Key words. uncertainty quantification, error model, composition law, Riemann problem

AMS subject classifications. 65Z05, 65G99, 76L05

DOI. $10.1137 / \mathrm{S} 1064827503427534$

1. Introduction. We have developed an approach to uncertainty quantification to numerical simulations that is based on three ideas:

1. A combined approach for forward as well as inverse propagation of uncertainty $[12,13]$. This combined approach is important when the use of disparate sources of data, including data pertaining to observations of full system performance, is important.

2. Error models for numerical solution errors $[9,8,10,6]$. For multiscale problems and complex multiphysics problems, underresolved simulations and accompanying simulation error are frequently unavoidable in practice.

3. Parameterization, comprehensibility, and validation of simulation error models [7]. This step allows testing and validation to occur in somewhat idealized situations, less complex than the full system simulations but still applicable to them.

Our principal concern here is to develop a method for determining solution errors in shock wave interaction problems having a significant degree of complexity. Our

${ }^{*}$ Received by the editors May 8, 2003; accepted for publication (in revised form) February 18, 2004; published electronically DATE. The U.S. Government retains a nonexclusive, royalty-free license to publish or reproduce the published form of this contribution, or allow others to do so, for U.S. Government purposes. Copyright is owned by SIAM to the extent not limited by these rights.

http://www.siam.org/journals/sisc/x-x/42753.html

$\dagger$ Department of Applied Mathematics and Statistics, University at Stony Brook, Stony Brook, NY 11794-3600 and Center for Data Intensive Computing, Brookhaven National Laboratory, Upton, NY 11793 (glimm@ams.sunysb.edu). Supported in part by the NSF Grant DMS-0102480 and the U.S. Department of Energy Grant DE-AC02-98CH10886 and the Los Alamos National Laboratory, Contract 26730001014L and the Army Research Office Grant DAAD-190110642 and the U.S. Department of Energy Grants, DE-FG03-98DP00206 and DE-FG02-90ER25084.

${ }_{\ddagger}^{\ddagger}$ Los Alamos National Laboratory, Los Alamos, NM 87545 (jgrove@lanl.gov, dhs@t13.lanl.gov). The second and sixth authors were supported by the U.S. Department of Energy. The third author was supported in part by the NSF Grant DMS-0102480 and the U.S. Department of Energy Grant DE-AC02-98CH10886 and the Los Alamos National Laboratory, Contract 26730001014L.

$\S$ Department of Applied Mathematics and Statistics, University at Stony Brook, Stony Brook, NY 11794-3600 (twlee@ams.sunysb.edu, linli@ams.sunysb.edu, yan2000@ams.sunysb.edu, kye@ams. sunysb.edu, mingzhao@ams.sunysb.edu). The fifth author was supported in part by the Army Research Office Grant DAAD-190110642 and the U.S. Department of Energy Grants, DE-FG0398DP00206 and DE-FG02-90ER25084 and U.S. Department of Energy Grant DEFC02-01ER25461. 


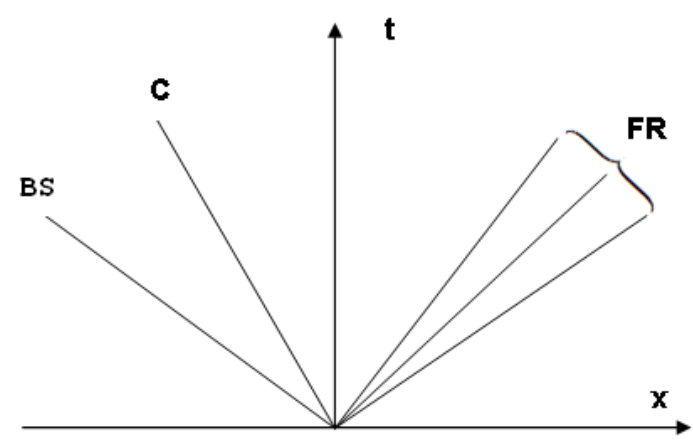

FIG. 1.1. Schematic diagram illustrating the solution of a Riemann problem. The solution contains (from left to right) a backward shock wave (labeled BS), a contact discontinuity (labeled C), and a forward rarefaction wave (labeled FR).

strategy takes advantage of the fact that shock problems typically consist of smooth regions separated by discontinuities (actually narrow regions with strong solution gradients), and it consists of three main steps.

Step 1: Determine solution errors for a comprehensive set of elementary wave interactions. These are summarized as a set of input/output relations for the errors in such interactions.

Step 2: Construct wave filters that decompose a complex flow into approximately independent components consisting of elementary waves.

Step 3: Formulate a composition law that constructs the total solution error at any space-time point in terms of errors from repeated elementary interactions.

Step 1 is carried out in sections 2 and 3. Step 2 is discussed in [16]. The main idea is summarized and extended in section 3 . The composition law is presented in section 4 .

The results are derived by a study on errors in the solution of Riemann problems. In section 2 we study the statistical Riemann problem (SRP). The Riemann problem is a simple jump discontinuity between two hydrodynamic states in one spatial dimension; its idealized solution is called the Riemann solution, as shown in Figure 1.1 and in [3]. With the inclusion of the nonlocalized rarefaction and compression waves in our discussion, the Riemann solution becomes an approximate model to the true interaction of these waves and in fact represents the leading asymptotes for the large time solution. We are specifically interested in states describing two incoming waves. In this sense we specialize the Riemann problem to a scattering problem or S-matrix problem [11]. But we study this S-matrix scattering problem from a statistical point of view. The SRP maps a given distribution of input wave strengths (to parameterize the two incoming waves about to interact) to an output distribution of wave strengths and speeds, and can be viewed as a nonlinear map from input data to output data. With our S-matrix emphasis, the input state data consists of one starting state (three parameters) and two wave strengths. The output data consists of the same starting state and three wave strength parameters. Thus the S-matrix Riemann problem is a map from $R^{2}$ to $R^{3}$ depending parametrically on $\theta \in R^{3}$. Our first objective is to analyze this problem statistically.

Our approach to numerical solution errors is motivated by needs of uncertainty quantification. Specifically the Bayesian likelihood is (up to normalization) a proba- 
bility, which specifies probability of occurrence of an error of any given size. Unlike other authors $[15,2,17,5]$, who usually use observational errors or expert opinions to form the probability model for the likelihood, our approach is to use solution error models for the likelihood, which provides more scientific basis. The authors are not aware of comparable error analysis studies, but, of course, numerical simulation errors have been long studied from different points of view. The method of a posteriori analysis aims to construct an upper bound on the solution error, either theoretically validated or based on numerical experiments $[1,19,18,4]$. This method has been difficult to apply to nonlinear hyperbolic systems and in any case does not answer the questions addressed here. The method of asymptotic analysis of numerical solution errors is so old and well established that it is difficult to cite its origins [20]. We are primarily interested in errors in a preasymptotic range, so while these methods provide a theoretical framework which is valuable to our analysis, they do not provide the detailed estimates which we require. In any case our use of wave filters to diagnosis error leads to more precise measures of error than is normally considered in the asymptotic analysis.

In section 3 we study the statistical numerical Riemann problem (SNRP). This differs from the SRP in that incoming shock waves, specified numerically, have finite width determined from numerical as well as physical considerations. We note that incoming rarefaction and compression waves have physical time-dependent widths. It also differs in that the numerical algorithm that solves the Riemann problem creates (as well as propagates) errors. We will show that, to fairly good accuracy, one can model the errors in the outgoing waves as affine linear, i.e., constant plus linear (or perhaps bilinear) statistical expressions in the strength of the incoming waves. The coefficients in these linear expressions depend parametrically on the incoming waves, here taken as defined by a base case characteristic of the ensemble as a whole.

Simulation errors typically consist of

- position errors in the location of the traveling wave discontinuities or sharp solution gradients;

- wave width errors in the numerical versus the physical width of the traveling waves;

- solution state errors in the smooth regions bordering the regions with discontinuities or sharp solution gradients.

Any or all or these errors may arise in the input data to the Riemann problem. Output errors, however, have two sources. Those arising from inaccuracies in the solution algorithm are called created errors, while those that can be ascribed directly to input error or uncertainty are called transmitted errors or transmitted uncertainty.

In section 4 we study the composition of these Riemann problems. We introduce a complex one-dimensional shock wave interaction problem, generated by a shock wave interacting with a contact in the vicinity of a wall. Multiple reflections between the wall and contact generate a large number of Riemann problems, which comprise the major features of this problem. The errors can be computed in two ways. First, we compute the errors directly by statistical analysis of the data for complex, multi-interaction simulations, comparing fine and coarse grid simulations. Second, we propose and implement a multipath integral formula for combining the errors created by and propagated through the individual Riemann problems and thereby predict the errors in the composite (complex) simulation. The comparison of errors for the complex, multi-interaction problem, thus determined in two ways, provides validation for our proposed composition law for errors and is the main result of this paper. 
2. The statistical Riemann problem. The main result of this section, perhaps a bit surprising, is that a linear input-output model will serve to describe the manifestly nonlinear problem of the interaction of strong hydrodynamic waves.

In this section, the numerical integrator (solution operator) is considered as exact and acts to propagate error (or uncertainty) in the input to error (or uncertainty) in the output. The output errors can be represented conceptually as a multinomial in powers of the strengths of the waves that are interacting. We characterize the statistics of the error as Gaussian, and thus it is determined by its mean and covariance. For weak waves these quantities, as a description of the input, are transformed via the solution operator Jacobean to the mean and covariance of the output. We are more interested in strong waves but find that a linear description of the solution operator still provides a useful description of the input to output transformed error statistics.

2.1. A multinomial expansion for the SRP output. The SRP has two incoming waves. Each may be one of the following: contact, shock, rarefaction, compression. Of course for incoming rarefaction and compression waves, the initial value problem for the interaction is not self-similar and thus does not strictly define a Riemann problem. In such cases we model the interaction using the Riemann problem with data given by the states behind the rarefaction of compression wave. For the pairwise interactions, we distinguish (for shock interactions with other shocks, with rarefactions, and with compression waves) whether the two interacting waves are of the same (left moving versus right moving) family or opposite families. For a contact wave, we specify the direction of the density change (step up or down) as seen from the side of the approaching wave. Thus there are a total of 14 elementary cases for the incoming waves. Rarefactions and compression waves interact only when they belong to opposite families, while two contacts never interact. Each case has associated with it a wave amplitude of a specific sign, so that there is no loss of generality in assuming that the signs are chosen so that the amplitudes are nonnegative. Fixing one case, we specify a starting state for the incoming waves and a base case for the (strong) wave strengths. Then we consider variation about this base case by $\pm 1 \%, \pm 10 \%$, and $\pm 50 \%$ in the density ratio (for contacts) or pressure (for all other waves). Within this formulation, we can describe the output wave strengths by an expression linear in the two input wave strengths, with a possible additional bilinear term, i.e., linear in the product of the input wave strengths. The linear and bilinear coefficients in this representation depend parametrically on the starting state.

The range of validity of such a bilinearization will depend on details in the problem formulation. Perhaps the most important of these is the choice of variables for the description of the wave strengths. We choose dimensionless variables to measure these strengths, a modified Atwood number $A=\left(\rho_{2}-\rho_{1}\right) /\left(\rho_{2}^{0}+\rho_{1}^{0}\right)$ to measure the contact strengths, and a similar expression built out of the pressures, $P=\left(P_{2}-P_{1}\right) /\left(P_{2}^{0}+P_{1}^{0}\right)$, for the other wave types. Here the quantities $\rho_{i}^{0}$ and $P_{i}^{0}$ denote densities and pressures from the base case associated with the ensemble. Of course the SRP, being fully nonlinear, will require an infinite Taylor's series for its complete description. Depending on the size of the statistical study, all terms in the series expansion will be observable. For a given finite sample size, one can ask whether certain coefficients in the expansion can be set to zero, in the sense that the observed nonzero value could result from the finite sample size. For each term we can introduce a hypothesis that the true expansion is missing this term (has a coefficient of zero) and assign a probability (p-value) to this hypothesis. On this basis we find that many terms are required to describe the statistics, and more would be needed if the sample size were larger. 
However, this statistical test misses the point and the value of a simple model. We want to capture the main effects and regard the remainder not as a statistical sampling error but as a modeling error. Thus we consider an expansion with more terms than we ultimately want and discard terms with small coefficients even if the associated p-value is not near 1 . For input wave strengths $w_{1}^{i}, w_{2}^{i}(i \equiv$ in) and output wave strengths $w_{1}^{o}, w_{2}^{o}$, and $w_{3}^{o}$ (o $\equiv$ out) (ordered from left to right), the expansion is defined by its coefficients $\alpha_{k, J}$ for $J$ a multi-index, $J=\left(j_{1}, j_{2}\right)$. The expansion has the form

$$
w_{k}^{o}=\sum_{J} \alpha_{k, J} w^{i, J},
$$

where $w^{i, J}=\left(w_{1}^{i}\right)^{j_{1}}\left(w_{2}^{i}\right)^{j_{2}}$. The coefficients $\alpha_{k, J}$ depend parametrically on the base case Riemann problem, about which a specified variation is allowed. Given a statistical ensemble of input and output values $w^{i}$ and $w^{o}$, we use a least squares algorithm to determine the best fitting model parameters $\alpha_{k, J}$ for any given polynomial order of model. We use (2.1) variationally, that is, to map input variation (about the base case for the ensemble) to output variability. In other words, (2.1), which is a formula for wave strengths, implies a similar formula with different but computable coefficients $\alpha_{k, J}$, in which all $\omega$ 's are defined as variations from the base case, so that they represent uncertainty or error. For simplicity we consider the case of a linear inputoutput relation, $\omega_{k}^{o}=\alpha_{k, 0}+\sum_{j} \alpha_{k, j} \omega^{i, j}$. If we denote the base case with an overbar, i.e., $\bar{\omega}_{k}^{o}$ and $\bar{\omega}^{i, j}$, then the fluctuation $\delta \omega_{k}^{o}=\omega_{k}^{o}-\bar{\omega}_{k}^{o}$ satisfies $\delta \omega_{k}^{o}=\sum_{j} \alpha_{k, j} \delta \omega^{i, j}$.

2.2. Evaluation of the expansion coefficients. The main point of this section is to give numerical values to the expansion coefficients for the output wave strengths of the SRP. We also find that for many cases, the linear model is sufficient for scientific accuracy, while the case of large variability will likely require additional expansion terms. Here we find that bilinear terms are sufficient for large variability.

We consider as a typical Riemann problem, the wave interaction of a shock wave moving (to the right) into a contact (density increase, or step up case). This wave interaction initiates the complex series of interactions studied in section 4 . The base case shock strength is given with a pressure ratio $1337(P=0.999)$ and contact density ratio $10(A=0.82)$. The equation of state is a gamma law gas, with $\gamma=1.67$.

Typical results are presented in Table 2.1 (variation of the polynomial order of the model) and Table 2.2 (variation of the strength of the input waves). To read these tables, we note that the first $\left(w_{1}^{o}\right)$ row of Table 2.1 (labeled in the table as $w_{1}^{o}$ (l. sonic)) lists coefficients $\alpha_{1, J}$ for $J=(0,0), J=(1,0)$, etc. These coefficients are determined by a least squares algorithm, that minimizes the expected, or mean error over the ensemble, in comparing the linear (or bilinear, or quadratic) predictions to the exact solution of the Riemann problem. The mean is taken over the statistical ensemble. The last two columns describe errors in model (2.1). The column labeled $L_{\infty}$ is the maximum of the absolute value, over the ensemble, of the relative error, expressed in percent. It is thus a pointwise error estimate. The maximum is computed using a sample size of 200 , and it may be sensitive to the choice of ensemble. The relative error is defined as (predicted-exact)/exact where exact is the result of the (exact) Riemann solution and predicted is the value given by the finite polynomial (linear, etc.) model. The column STD is the standard deviation of (predicted-exact). From the small values of these errors for the linear model, as seen in Table 2.1, we conclude that the linear model is adequate for many purposes. The $4 \times 3$ bilinear subblock of the quadratic model is nearly identical to the bilinear model, and the 
TABLE 2.1

The shock-contact (step up) interaction. Expansion coefficients for output wave strengths for input variation $\pm 10 \%$. Comparison of linear, bilinear, and full quadratic models for the output variables $w_{k}^{o}$.

\begin{tabular}{|c|c|c|c|c|c|c|c|c|}
\hline \multirow[t]{2}{*}{ Variable $\backslash$ Coef } & \multirow[t]{2}{*}{ Const } & \multirow{2}{*}{$\begin{array}{c}\quad w_{1}^{i} \\
\text { (r. sonic) } \\
\end{array}$} & \multirow{2}{*}{$\begin{array}{c}w_{2}^{i} \\
\text { (contact) }\end{array}$} & \multirow{2}{*}{$w_{1}^{i} w_{2}^{i}$} & \multirow{2}{*}{$\left(w_{1}^{i}\right)^{2}$} & \multirow{2}{*}{$\left(w_{2}^{i}\right)^{2}$} & \multicolumn{2}{|c|}{ Error } \\
\hline & & & & & & & $L_{\infty}$ & STD \\
\hline \multicolumn{9}{|c|}{ Linear response model } \\
\hline$w_{1}^{o}$ (l. sonic) & -0.206 & 0.452 & 0.252 & & & & $0.55 \%$ & 0.0008 \\
\hline$w_{2}^{o}($ contact $)$ & -0.042 & 0.000 & 0.911 & & & & $0.01 \%$ & 0.00004 \\
\hline$w_{3}^{o}(\mathrm{r}$. sonic $)$ & -0.285 & 1.001 & 0.347 & & & & $0.35 \%$ & 0.001 \\
\hline \multicolumn{9}{|c|}{ Bilinear response model } \\
\hline$w_{1}^{o}$ (l. sonic) & 0.011 & 0.234 & -0.015 & 0.267 & & & $0.16 \%$ & 0.0003 \\
\hline$w_{2}^{o}$ (contact) & -0.043 & 0.001 & 0.912 & -0.001 & & & $0.01 \%$ & 0.00004 \\
\hline$w_{3}^{o}$ (r. sonic) & 0.015 & 0.701 & -0.020 & 0.368 & & & $0.10 \%$ & 0.01 \\
\hline \multicolumn{9}{|c|}{ Quadratic response model } \\
\hline$w_{1}^{o}$ (1. sonic) & -0.090 & 0.245 & 0.222 & 0.253 & 0.000 & -0.136 & $0.01 \%$ & 0.00002 \\
\hline$w_{2}^{o}$ (contact) & -0.030 & 0.001 & 0.881 & 0.001 & 0.000 & 0.018 & $0.00 \%$ & $1.1 \mathrm{E}-6$ \\
\hline$w_{3}^{o}(\mathrm{r}$. sonic $)$ & -0.125 & 0.715 & 0.305 & 0.349 & 0.001 & -0.187 & $0.02 \%$ & 0.00002 \\
\hline
\end{tabular}

TABLE 2.2

The shock-contact (step up) interaction. Expansion coefficients for output wave strengths. Comparison of input variation $( \pm 1 \%, \pm 10 \%, \pm 50 \%)$. The linear model is used for the first two cases, while the bilinear model is required for the largest variation.

\begin{tabular}{|c|c|c|c|c|c|c|}
\hline \multirow[t]{2}{*}{ Variable $\backslash$ Coef } & \multirow[t]{2}{*}{ Const } & \multirow{2}{*}{$\begin{array}{l}\quad w_{1}^{i} \\
\text { (r. sonic) }\end{array}$} & \multirow{2}{*}{$\begin{array}{c}w_{2}^{i} \\
\text { (contact) }\end{array}$} & \multirow[t]{2}{*}{$w_{1}^{i} w_{2}^{i}$} & \multicolumn{2}{|c|}{ Error } \\
\hline & & & & & $L_{\infty}$ & STD \\
\hline \multicolumn{7}{|c|}{$1 \%$ input variation; linear model } \\
\hline$w_{1}^{o}$ (l. sonic) & -0.207 & 0.453 & 0.252 & & $0.01 \%$ & $8.0 \mathrm{E}-6$ \\
\hline$w_{2}^{o}($ contact $)$ & -0.042 & 0.000 & 0.911 & & $0.00 \%$ & $3.6 \mathrm{E}-7$ \\
\hline$w_{3}^{o}(\mathrm{r}$. sonic $)$ & -0.285 & 1.002 & 0.347 & & $0.01 \%$ & 0.0001 \\
\hline \multicolumn{7}{|c|}{$10 \%$ input variation; linear model } \\
\hline$w_{1}^{o}$ (l. sonic) & -0.206 & 0.452 & 0.252 & & $0.55 \%$ & 0.0008 \\
\hline$w_{2}^{o}$ (contact) & -0.042 & 0.000 & 0.911 & & $0.01 \%$ & 0.00004 \\
\hline$w_{3}^{o}(\mathrm{r}$. sonic $)$ & -0.285 & 1.001 & 0.347 & & $0.35 \%$ & 0.001 \\
\hline \multicolumn{7}{|c|}{$50 \%$ input variation; bilinear model } \\
\hline$w_{1}^{o}$ (l. sonic) & 0.013 & 0.216 & -0.016 & 0.280 & $4.15 \%$ & 0.008 \\
\hline$w_{2}^{o}$ (contact) & -0.041 & 0.002 & 0.911 & -0.001 & $0.89 \%$ & 0.001 \\
\hline$w_{3}^{o}(\mathrm{r}$. sonic $)$ & 0.017 & 0.676 & -0.022 & 0.385 & $4.15 \%$ & 0.116 \\
\hline
\end{tabular}

two pure quadratic columns of the quadratic model are small, indicating that the quadratic model is basically a correction to the bilinear model. However, the $3 \times 3$ linear subblock of the bilinear model is not a good approximation to the corresponding values of the linear model. Moreover, in this case the size of the pure bilinear terms in the model is large relative to the decrease in error achieved by use of the bilinear model relative to the linear one. Thus we conclude that the bilinear model contains a substantial amount of cancellation between its terms, and in general the linear model may be more satisfactory. All three models in Table 2.1 show an approximate diagonal, identity matrix structure for the $2 \times 2$ subsystem formed by the right sonic and contact waves, as would be expected from a linear (small amplitude) theory. Observe that the approximate validity of a linear model has implications for the statistics, as linear transformations map Gaussian statistics into Gaussian statistics. 
TABLE 2.3

The shock crossing-equal shock (wall reflection) interaction. Expansion coefficients for output wave strengths (linear model) for input variation $\pm 10 \%$.

\begin{tabular}{|lllll|}
\hline Variable \Coef & Const & \multicolumn{2}{c|}{$w_{1}^{i}$} & \multicolumn{2}{c|}{ Error } \\
& & (r. sonic) & $L_{\infty}$ & STD \\
\hline$w_{1}^{o}$ (l. sonic) & -0.0008 & 0.715 & $0.00 \%$ & $4 \mathrm{E}-8$ \\
\hline
\end{tabular}

From Table 2.2 we see that the model coefficients show only a slight sensitivity to the magnitude of fluctuation of the input variables. The small (1\% and $10 \%)$ fluctuation models have coefficients computable from linear theory, but these coefficients do not define the best coefficients for the larger fluctuation models. The linear model is not acceptable for the larger (50\%) variation, so we use the bilinear model in that case. Surprisingly, the errors for the quadratic model with $50 \%$ variation are somewhat higher than for the bilinear. The least squares algorithm used to define the model coefficients does not optimize them relative to the sup norm errors reported in the tables.

In Table 2.3 we consider the shock crossing-shock case. The two waves are of opposite families, and each has Mach number 53. This case is also the second (in time) Riemann problem to occur within the complex problem studied in section 4 as the shock reflection from a wall.

2.3. Variance. Here we introduce the important issue of the correlation among the output waves. In essence our analysis will proceed by ignoring this correlation. Having done so, we determine the variance of the output waves as a function of the input distributions. The formulas are first generally applicable and then specialized to our specific situation, including the assumption of a linear input-output model, in which case they are standard.

The Riemann solution $w^{o}=W^{o}\left(w^{i}\right)$ defines a mapping from the input to the output statistics. The approximate mapping (2.1) defines an easily computable approximation to this transformation of statistics. Thus for a given probability distribution $d \nu^{i}\left(w^{i}\right)$ defined on the input variables and supported in a domain $\mathcal{D}_{i}$ of input variables, the output variables lie in a domain $\mathcal{D}_{o}$, and on this domain the probability measure for the output variables is

$$
d \nu^{o}\left(w^{o}\right)=\frac{d \nu^{o}\left(W^{o}\right)}{d \nu^{i}\left(w^{i}\right)} d \nu^{i}\left(\omega^{i}\right)
$$

From the above formulas, we see an immediate problem. The three output variables, defined as a function of the two input variables, cannot be statistically independent. They lie in a two-dimensional subdomain, $\mathcal{D}_{o}$, of the three-dimensional space of output wave parameters. However, these waves separate and to a large extent carry out separate roles in the composite wave interaction problem studied in section 4 . Thus we want to generate independent statistical descriptions of each of the output waves. This step, of ignoring the correlations in the statistics of the outgoing waves, assumes that the successive interactions give rise to a decorrelation of the solution from its dependence on the earlier interactions. In a strict sense this assumption is not correct, but we believe it will give a reasonably accurate description of the final composite statistics. The validity of this assumption will be tested in the comparison given in section 4, where composition of errors from interacting Riemann problems are considered.

We next deal with the assumptions of input independence and output variance in a quantitative manner. We write the domain $\mathcal{D}_{o}$,

$$
\mathcal{D}_{o} \subseteq \mathcal{D}_{o}^{1} \times \mathcal{D}_{o}^{2} \times \mathcal{D}_{o}^{3},
$$


as a subset of its projections $\mathcal{D}_{o}^{k}$ on the three output wave strength coordinate axes. The marginal distribution is the projection of the three-dimensional measure $d \nu^{o}$ achieved through its integration over the two neglected variables. Assuming sufficient regularity, the marginal distribution can be written as

$$
d \nu_{k}^{o}\left(w_{k}^{o}\right)=\frac{d \nu_{k}^{o}}{d w_{k}^{o}} d w_{k}^{o}=\int_{\mathcal{D}_{o}\left(w_{k}^{o}, w_{k}^{o}+d w_{k}^{o}\right)} d \nu^{o}\left(w^{o}\right),
$$

where

$$
\mathcal{D}_{o}\left(w_{k}^{o}, w_{k}^{o \prime}\right)=\mathcal{D}_{o} \cap R^{2} \times\left[w_{k}^{o}, w_{k}^{o \prime}\right] .
$$

Let us suppose that the distribution of the incoming waves $d \nu^{i}$ is absolutely continuous and that it is a product of the individual measures on the incoming waves. (Thus the incoming waves are assumed to be statistically uncorrelated. Note that they are also output waves of other (but distinct) Riemann problems.) In this case,

$$
d \nu^{i}=f_{1}^{i}\left(w_{1}^{i}\right) f_{2}^{i}\left(w_{2}^{i}\right) d w_{1}^{i} d w_{2}^{i}
$$

for pdfs $f_{k}^{i}$. It is convenient to introduce the cumulative distribution function $F^{i}$ so that

$$
d \nu^{i}=d F^{i}=f_{1}^{i}\left(w_{1}^{i}\right) f_{2}^{i}\left(w_{2}^{i}\right) d w_{1}^{i} d w_{2}^{i}
$$

and the marginal cumulative distribution functions $F_{j}^{o}$ for which

$$
F_{j}^{o}\left(w_{j}^{o}\right)=\int_{\left\{W_{j}^{o}\left(w_{1}^{i}, w_{2}^{i}\right)<w_{j}^{o}\right\}} d F^{i}=\int_{\left\{W_{j}^{o}\left(w_{1}^{i}, w_{2}^{i}\right)<w_{j}^{o}\right\}} f_{1}^{i}\left(w_{1}^{i}\right) f_{2}^{i}\left(w_{2}^{i}\right) d w_{1}^{i} d w_{2}^{i} .
$$

We note that

$$
f_{j}^{o}\left(w_{j}^{o}\right)=\frac{d F_{j}^{o}\left(w_{j}^{o}\right)}{d w_{j}^{o}} .
$$

Next we assume a linear model

$$
w_{j}^{o}=\alpha_{j}+\sum_{k=1}^{2} \beta_{j k}^{i} w_{k}^{i}
$$

for the SRP. We can now compute the marginal cumulative distribution functions explicitly in terms of the coefficients for the linear model for the SRP,

$$
\begin{aligned}
& F_{j}^{o}\left(w_{j}^{o}\right)=\int_{-\infty}^{\infty} \int_{-\infty}^{\left(w_{j}^{o}-\alpha_{j}-\beta_{j 2}^{i} w_{2}^{i}\right) / \beta_{j 1}} f_{1}^{i}\left(w_{1}^{i}\right) f^{i}\left(w_{2}^{i}\right) d w_{1}^{i} d w_{2}^{i}, \\
& f_{j}^{o}\left(w_{j}^{o}\right)=\int_{-\infty}^{\infty} \frac{1}{\beta_{j 1}^{i}} f_{1}^{i}\left(\left(w_{j}^{o}-\alpha_{j}-\beta_{j 2}^{i} w_{2}^{i}\right) / \beta_{j 1}^{i}\right) f_{2}^{i}\left(w_{2}^{i}\right) d w_{2}^{i} .
\end{aligned}
$$

Assuming statistical independence of the $w_{k}^{i}$ as above, a simple calculation shows that the variance, $\operatorname{Var} w_{j}^{o}$, equals

$$
\operatorname{Var} w_{j}^{o}=\sum_{k=1}^{2}\left(\beta_{j k}^{i}\right)^{2} \operatorname{Var} w_{k}^{i} .
$$

Using (2.13) and information such as that tabulated in Tables 2.1 and 2.2 to generate the $\beta$ 's, we can predict the propagation of uncertainty through a series of wave interactions; see section 4 . 
3. The statistical numerical Riemann problem. The SNRP has a new feature beyond those of the SRP studied in section 2. It introduces errors (modeled as random) in addition to propagating errors or uncertainty from input to output. The waves in the SNRP have a finite width, and the solution algorithm in the SNRP has only finite accuracy. Because of the possible finite width to the input waves, the problem and its solution are not strictly scale invariant, and so we consider a generalization of the Riemann problem.

3.1. The wave filter. We introduce diagnostic windows, which measure the solution state in one of the constant regions between the waves as well as wave filters, that diagnose the wave type (only regions with a single wave pass through the filter). In doing so, we first summarize and then extend the results of [16]. The moving window in the wave filter has an initial width of 5 cells for shock and rarefaction waves and 11 cells for contacts. The choice of these parameters appears to be suitable for most higher-order Godunov schemes. In this window, a Riemann problem is solved using the extreme left and right states as input. The Riemann solution has three outgoing waves, whose strength is assessed dimensionlessly as in section 2 in terms of density and pressure differences and ratios. According to these strengths and a suitable cutoff for the strength, we identify from zero to three of the waves as strong, and only the case of a single strong wave is analyzed further. If adjacent or overlapping windows show a single identical wave, the windows are merged, so that the full width of the wave will be brought into a single window. This merging of adjacent or overlapping windows of increasing size continues recursively until the same wave type fails to show up in the adjacent windows. Wave profiles are reconstructed using fitting functions of the form

$$
\rho(x, t)=\rho_{-}+\frac{\rho_{-}-\rho_{+}}{2}\left(f\left(\frac{x-x_{c}(t)}{\sqrt{2} \sigma}\right)+1\right),
$$

where $\rho_{ \pm}$refer to the asymptotic values for density ahead or behind the wave, $x_{c}(t)$ is the moving center of the wave, and $2 \sigma$ is a measure of the wave width. The fitting function $f(x)$ is either the erf function

$$
f(x)=\operatorname{erf}(x)=\frac{2}{\sqrt{\pi}} \int_{0}^{x} e^{-t^{2}} d t
$$

for contact or shock waves, or a linear ramp

$$
f(x)=\left\{\begin{aligned}
-1, & x<-1, \\
x, & -1<x<1, \\
1, & 1<x
\end{aligned}\right.
$$

for rarefactions and compressions. The four fitting parameters are determined by solving the nonlinear least squares problem for the data in the interval found by the Riemann problem filter described above. States identified as within an active region for a single shock or contact wave by the filter are fit to an error function, which allows determination of the location of the wave (with subgrid accuracy, up to $\mathcal{O}\left(\Delta x^{2}\right)$ ), and its width; see Figure 3.1 (left). For single rarefaction or compression waves, the waves are fit to a straight line segment, linear in the characteristic speed variable. Thus the region of constant and variable states are fit to three straight line segments, the two extreme ones being constants; see Figure 3.1 (right). The width for a shock or contact wave is defined in terms of the error function fit to the sock or contact wave 

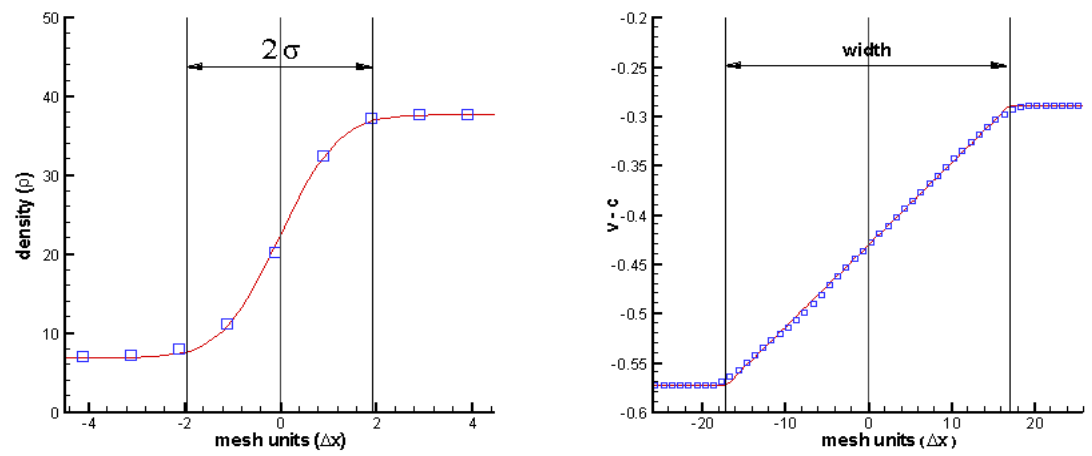

FIG. 3.1. Schematic diagram illustrating the operation of a wave filter. Left: computational data (squares) are fit to an error function. The error function depends on four parameters: a position, a width, and two asymptotic values. These determine the wave position, width and height, with subgrid accuracy. Right: a piecewise linear construction is fit to the rarefaction or compression wave data.

profile. Let $\sigma$ be the standard deviation that enters into the definition of the error function. Then the width (in units of $\Delta x$ ) is the distance needed for a $2 \sigma$ transition (between 2.3\% and 97.7\%) of the jump in the density (for a contact wave) or in the pressure (for a shock wave). The width of a rarefaction or compression wave is defined as the distance between the edges of the central linear piece for its piecewise linear description. The position is defined, with subgrid accuracy, as the position of the mean value, at a point half way through the jump. In this way we record the left and right states, wave position (and hence speed), and wave width. These quantities are not independent, as the speeds and jumps are connected by the Rankine-Hugoniot jump relations. They are sufficient to fully characterize the numerical incoming waves. The location where the linear fit attains the far field state is marked as the edge of the wave, and its width is the distance between its two edges. Finally, the filter regards any shock-like wave that is "too wide" in mesh units to be a compression wave.

The wave filter is the fundamental diagnostic tool that identifies individual waves, here within the solution of a numerical Riemann problem and in section 4 within the solution of a complex wave interaction problem. We note immediately a limitation of the methodology, at least as presently developed. The definition of the wave filters assumes that the individual waves in the Riemann problem have separated. For sufficiently coarse grids in the wave interaction problem of section 4 , the waves will enter into new interactions before clearly separating as they leave an earlier interaction. A second and related limitation concerns the relaxation of the left and right states at the edge of a wave to their far field values, an issue studied in section 3.2. If a subsequent interaction occurs before this relaxation is complete, the associated errors will be "frozen" into the input and output of this later interaction. We will assess this issue in section 4 .

A statistical distribution of numerical incoming waves and starting states determines the SNRP. Its solution gives the output waves, each of which generates the same type of data. Thus we define the SNRP as a statistical (nondeterministic) mapping from a statistical input wave description to a statistical output wave description.

The statistics of the SNRP mapping function arise from grid errors and from the random placement of a traveling wave relative to the centers of the finite difference 
lattice. Our first objective in this section is to compare and contrast the SRP and the SNRP. Our second objective is to build up a library of statistical input-output relations that will include all Riemann problems to be encountered in section 4 . This library will be used to predict results for the multiwave error and uncertainty analysis based on a multipath scattering formula.

3.2. The isolated wave. We start with the analysis of the ensemble averaged mean width of a single (noninteracting) wave. Figure 3.2 (left) shows the expected narrow and time-independent $(\sim 2 \Delta x)$ shock width. Among the several factors contributing to wave strength and speed errors, we mention the finite accuracy of the Riemann solution root solver, or some approximate Riemann solver, used in the numerical scheme, and the numerical (finite difference) nature of the solution. The latter arises in two ways: the relaxation to a constant ambient state and the finite rate of convergence under mesh refinement, both applicable on the postshock or up-stream side of the shock wave. The equivalence of these two effects can be seen from the self-similar nature of the Riemann solution, which implies that the fixed mesh, large time limit, with evaluation of the ambient state at a large separation from the traveling wave, is equivalent to a fixed separation at a fixed time, considered as a mesh refinement problem.

According to the theory of traveling waves for the viscous Riemann problem [21], considered as a model for numerically generated traveling waves, we expect an exponential approach of the numerical shock profile to its limiting values at $x= \pm \infty$. Depending on the numerical scheme, moreover, this approach may be oscillatory. For higher-order flux or slope limited methods there is a trade-off between the oscillations and the wave width, as the oscillations are reduced through increase of the first order (diffusive) aspect of the algorithm, while the wave width is reduced through
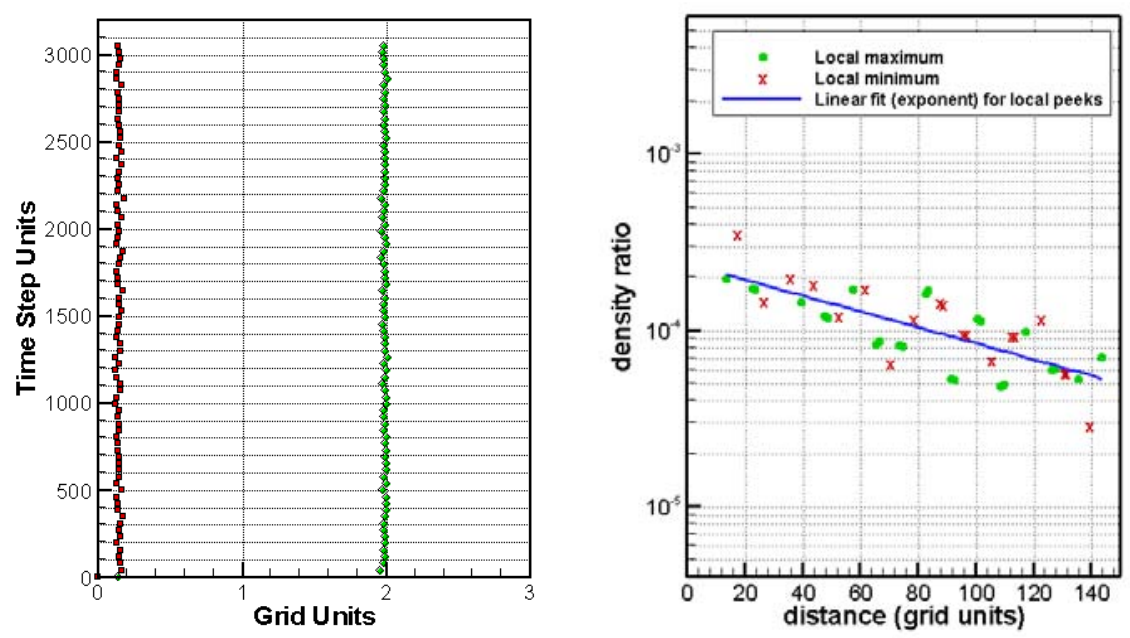

FIG. 3.2. Ensemble mean shock width and the standard deviation of the shock width (left frame). The mean width, equal to about $2 \Delta x$, is much larger than the standard deviation, indicating that the mean width is essentially a deterministic feature of the solution. Convergence properties of the traveling wave to the steady state values on each side of the wave (right frame). The straight line in the right frame is the asymptote to the exponential convergence rate, with slope 0.01 in units of $\Delta x$. 

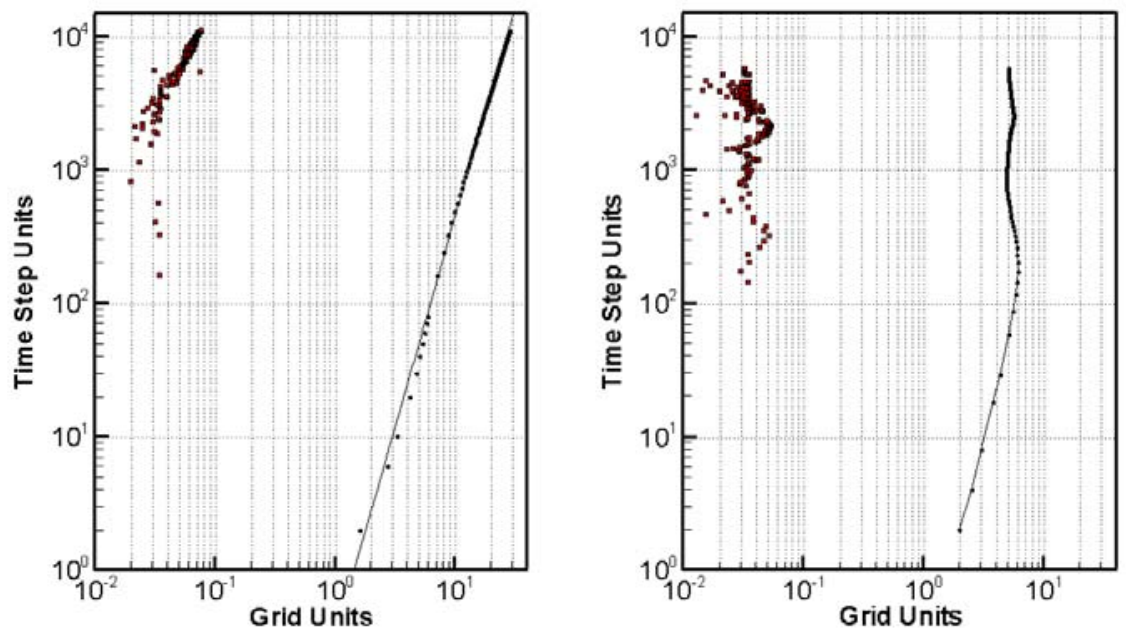

FIG. 3.3. Ensemble mean contact width for isolated noninteracting waves. Because the width is entirely grid related, we record width in units of $\Delta x$ and time in units of the number of time steps. The standard deviations are also plotted, and so are the points to the extreme left in each frame. Left (step down): we observe an increase from 2 cells to 30 over $10^{4}$ steps and an asymptotic growth rate $c_{c} t^{1 / 3}$, where $c_{c} \sim 1$ depends on the flow Mach number. The straight line in the left frame is the asymptote to the contact width, with slope 3 . Right (step up): we observe a bound on the contact width.

decrease in this diffusiveness. The error occurs on the upwind side of the shock, while the downwind states converge identically to their far field values within a few mesh blocks. See Figure 3.2 (right). We measure the local extrema in the error dimensionlessly as $e_{\max }=\left|\left(\rho-\rho_{\infty}\right) / \rho_{\infty}\right|$, where $\rho_{\infty}$ is the far field density. Then we model $e_{\max }(n \Delta x)=c \exp (-\lambda n)$, where $n$ is the distance from the shock front in mesh units. We find $\lambda=1.0 \times 10^{-2}$ and $c=2.4 \times 10^{-4}$ in the present case. The first extremum is a local maximum, occurring about $4 \Delta x$ from the center of the shock front. The details of the shock error behavior will be sensitive to the numerical method. We expect (but have not established) that the general form of the error, as it is derived from a mathematical theory, should be somewhat universal.

Figure 3.3 (left) shows the larger contact width $w_{c} \sim c_{c} t^{1 / 3}$ growing from 2 to 30 cells with a rate asymptotically proportional to $t^{1 / 3}$. Similar asymptotics have been observed by Harten [14] for an ENO scheme. The rate $t^{1 / 3}$ results from the second order accuracy of the method used here. The coefficient $c_{c}$ of the growth law is sensitive to the ambient Mach number $M$ of the flow, and more specifically to a transport CFL number $\theta=|v| /(|v|+c)=M /(M+1)$, assuming that the algorithm has a time step set by the CFL limit $\Delta t=\Delta x /(|v|+c)$. Here $c=\max \left\{c_{l}, c_{r}\right\}$ is the maximum of the left and right state sound speeds $c_{l}$ and $c_{r}$, respectively, and $v$ is the fluid velocity. The contact advances a fraction $\theta$ of one mesh cell in a single time step. For $\theta=0$ or $\theta=1$, the contact does not move in its relative position to the grid lines and $c_{c}=0.0$. This value is applicable for a very narrow range of theta. For most of the range of $\theta$, the numerical diffusion is sensitive to the direction of mixing. For $\theta<0.5$ and the flow from high density to low, the numerical mixing is that of heavy fluid into light. We call this the step down problem. For the step down problem, shown in Figure 3.3 (left), and for most $\theta$ values, we find $c_{c} \sim 1$, 
The reverse, called the step up problem, flows from light to heavy fluid. It mixes small amounts of light fluid into heavy fluid, an effect less noticeable in terms of the diffusion width, especially for large density contrasts. For a step up flow we find $w_{c} \sim \min \left\{5, c_{c} t^{1 / 3}\right\}$; see Figure 3.3. As a partial explanation of this difference between the step down and the step up problems, we note that the spreading is primarily associated with the up-stream side of the contact and that continued spreading (the $t^{1 / 3}$ asymptotics) depends on the up-stream flow being subsonic. The higher sound speed in the light fluid gives a supersonic up-stream state for the step up problem but not for the step down problem for the flow parameters considered here. These properties appear to be sensitive to the details of the numerical algorithm and specifically to the form of the limiter employed. We have used a MUSCL algorithm. The degree of recalibration of the model as presented here, needed for other solvers, is an important question, out of the scope of this study.

For some aspects of the solution error, the probabilistic error formalism is more general than is required. When the standard deviation of the error is much smaller than the mean error (when the coefficient of variation, their ratio, is close to zero), then the error is essentially deterministic, and the probabilistic formulation is unnecessary. The standard deviation of the width, shown to the right in each frame of Figure 3.3, is significantly smaller than the mean width, indicating that the wave width is essentially a deterministic feature of the numerical solution. The wave position errors have a statistical aspect, especially in their transient behavior.

3.3. Interactions with contact waves. We observe an increase in the contact width during the numerical shock-contact interaction process. In Figure 3.4 (left), we show the time development of the widths of the two incoming waves and the three outgoing waves for the shock-contact (step up) interaction. This interaction occurs early in the development of the contact. In Figure 3.4 (right) we show the
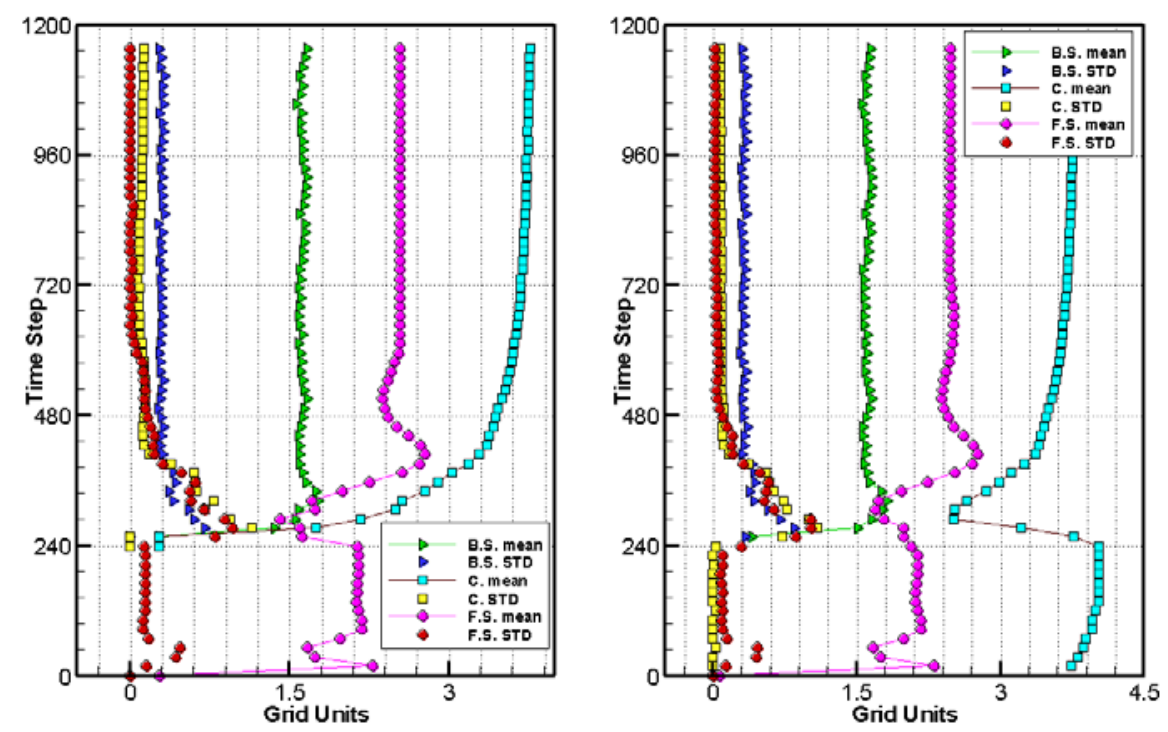

FIG. 3.4. Ensemble mean shock and contact widths before and after a shock-contact (step up) interaction and standard deviations. Left: interaction with a narrow contact (from early time). Right: interaction with a wide contact, as would occur for an interaction later in a simulation. 
same interaction but with a contact having a larger initial width, representative of a contact-shock interaction occurring late in a simulation. From this figure we see that while the transient contact width is sensitive to the input wave width, the large time asymptotic contact width is not.

We next study wave strength, speed, and position errors after a wave interaction. Note that the SNRP errors considered so far have no counterpart in the SRP.

We represent the wave properties as a quadruple

$$
w_{k}^{a}=\left(\omega_{k}^{a}, \lambda_{k}^{a}, s_{k}^{a}, p_{k}^{a}\right),
$$

where $\omega$, as in section 2, is a wave strength, $\lambda$ is a wave width, $s$ is a wave speed error, and $p$ is a position error. Also $a=i$ for input and $a=o$ for output. The units for wave strengths $A$ and $P$, as in section 2, are dimensionless. We measure the wave widths in units of mesh spacing, with the contacts having either a $t^{1 / 3}$ or bounded time dependence. Thus we interpret $\lambda_{2}^{i}$ as the input contact wave width in mesh units at the time of interaction (no time dependence) and $\lambda_{2}^{o} t^{1 / 3}$ or $\lambda_{2}^{o}$ as the output contact wave width, also in grid units, depending on the up-stream Mach number. Here $t$ is the elapsed time since the interaction, expressed in time step units. Because the shock width is deterministic and time-independent, it is not needed as an input or an output variable. The wave speed errors $s$ are specified dimensionlessly as a ratio (through division of the error by the exact speed value). The speed error $s$ occurs only as an output variable. This error refers to a far field value, after the waves are well separated. This large time asymptotic speed error is very small and will not be considered further. Near field state errors are given by an oscillatory decaying exponential, approaching the far field value. The exponent, or decay rate, characterizes this approach in a simple overall manner; see section 3.2. The wave position errors are specified in grid units, after an isolated transient period. We include these errors in the output description only, as input position errors correspond only to a shift in the location and time of an interaction and can be translated trivially to output position errors; see section 4.2.

The input multi-index $J=\left(j_{1}, j_{2}, j_{3}\right)$, with the above simplifications, now has three components while the output multi-index has six (to reflect the dependence on three position errors $p_{k}^{o}$ ). Using $J$, we define the power expressions

$$
w^{i, J}=\left(\omega_{1}^{i}\right)^{j_{1}}\left(\omega_{2}^{i}\right)^{j_{2}}\left(\lambda_{2}^{i}\right)^{j_{3}} .
$$

For the first three interactions we study, the input wave width $\left(\lambda_{2}^{i}\right)^{j_{3}}$ is not used, i.e., modeled as being zero. With these conventions, (2.1) also describes the model for the SNRP, but now each coefficient $\alpha_{k, J}$ is also a random variable, reflecting randomness in the numerical solution algorithm, such as the dependence of the solution on the subgrid location of the interaction point relative to the grid. The ensemble mean $\overline{\alpha_{k, J}}$ defines a model similar to that of section 2 but complicated by the additional indices and coefficients to reflect the errors $\lambda_{k}^{o}$ and $p_{k}^{o}$ (the variable $s_{k}^{o}$ can be suppressed). As in section 2, we can subtract the base case values from the $\omega_{k}^{i}$ and $\omega_{k}^{o}$ and then regard the $\omega$ (as with the other variables) as representing error or uncertainty.

We begin with the analysis of the SNRP at the ensemble averaged level. We present the mean model analysis in Table 3.1, for the same case as in Table 2.1, with $\pm 10 \%$ variation about the base case. We examine only the linear model. The input contact width has been set to zero, as part of the specification of this SNRP.

The first three rows of pure $\omega$ terms of Table 3.1 can be compared to the corresponding SRP values from Table 2.2. We note the near identity of the SRP and 
TABLE 3.1

The SNRP shock-contact (step up) interaction. Expansion coefficients for output wave strengths (linear model) for input variation $\pm 10 \%$. Here the base case input contact wave width is zero.

\begin{tabular}{|lrrrrr|}
\hline Variable $\backslash$ Coef & Const & \multicolumn{1}{c}{$\omega_{1}^{i}$} & \multicolumn{2}{c|}{$\omega_{2}^{i}$} & \multicolumn{2}{c|}{ Error } \\
& & (r. sonic) & (contact) & \multicolumn{1}{c}{$L_{\infty}$} & STD \\
\hline$\omega_{1}^{o}$ (l. sonic) & -0.208 & 0.454 & 0.251 & $0.47 \%$ & 0.001 \\
$\omega_{2}^{o}$ (contact) & -0.042 & 0.000 & 0.912 & $0.03 \%$ & 0.0001 \\
$\omega_{3}^{o}$ (r. sonic) & -0.286 & 1.004 & 0.346 & $0.30 \%$ & 0.001 \\
$\lambda_{1}^{o}$ (l. sonic) & 2.184 & -0.563 & 0.000 & $122 \%$ & 0.240 \\
$\lambda_{2}^{o}$ (contact) & 4.725 & 0.110 & -1.466 & $0.67 \%$ & 0.010 \\
$\lambda_{3}^{o}$ (r. sonic) & 2.197 & 0.068 & 0.106 & $5.35 \%$ & 0.057 \\
$p_{1}^{o}$ (l. sonic) & 0.221 & -0.014 & 0.023 & $27.1 \%$ & 0.022 \\
$p_{2}^{o}$ (contact) & 0.426 & 0.001 & -0.092 & $1.78 \%$ & 0.002 \\
$p_{3}^{o}$ (r. sonic) & 0.332 & -0.004 & -0.099 & $3.47 \%$ & 0.005 \\
\hline
\end{tabular}

SNRP values, indicating the numerical accuracy of the numerical scheme applied to Riemann data.

The three wave width variable $(\lambda)$ rows in Table 3.1 represent new errors relative to the SRP. The large sup norm error for the width of the reflected shock results from a few outliers, mostly but not entirely due to smaller shock widths than the mean. The standard deviation for this quantity is about $10 \%$ of the mean value, indicating that the error model is (on the whole) satisfactory and that the shock wave widths are not (mostly) fluctuating greatly. The outliers are mainly associated with time steps and realizations for which the (narrow) reflected shock has at most one internal mesh point. For these cases, our filter tool for assessing the numerical shock width and position is not effective, so the outliers can be viewed as a breakdown of the diagnosis methodology.

The shock speed errors are nearly zero (to 5 digit accuracy in relative shock speed errors) after separation of the interacting waves. For this reason these errors are not presented here. Consistent with the convergence properties of the traveling wave to its left and right states, shown in Figure 3.2, accurate determination of shock speed requires well-separated waves, and thus a highly resolved calculation.

We also study the wave position errors. It would be equivalent to study timing errors (for example, in wave arrival), since the position errors are the integral of the velocity errors. The fact that the position errors reach a steady nonzero asymptotic value is equivalent to the fact that the speed errors tend to zero, with a nonzero time-averaged value. Figure 3.5 shows the position errors as a function of time. In order to study the initial transients in the position errors systematically, we adjust the ensemble to have a common time and location of interaction. An initial transient error will be significant when we consider errors in underresolved simulations. Following this transient, the position error is constant, reflecting convergence of the wave speed to its exact value. The errors in the wave position rows of Table 3.1 present this constant error, given in mesh units. All position errors are subgrid. The standard deviations are smaller than the means, indicating that the errors are basically deterministic. The $L_{\infty}$ position error is the supremum of the relative error. It is an error in the model of the error, i.e., the error in the error. Occasional ensemble members with very small (exact) position error produce a small denominator in the relative error, (model error)/(exact error). Thus the large entries in this column (also in other tables) do not represent a deficiency in the error model. We see similar and more extreme $L_{\infty}$ percent errors in later tables, with the same cause. Note that the standard deviation 


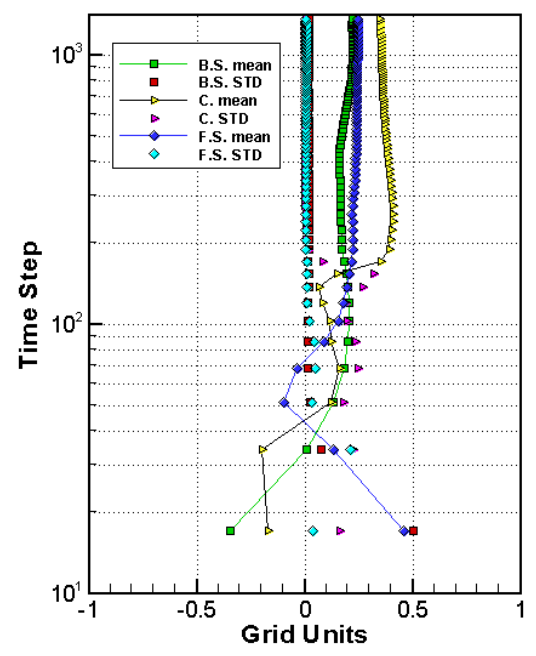

FIG. 3.5. Ensemble mean shock and contact position errors as a function of time, expressed in grid units (step up case).

is comparable to the mean position error, so that occasional instances of nearly zero error are to be expected.

Continuing with the study of position errors, we examine the shock crossing-shock and the shock crossing-contact (step down) cases, with an emphasis on the transient error; see Figure 3.6. The transient error is significant, on the order of the mesh spacing, and the standard deviation is also significant, in contrast to the steady or large time asymptotics emphasized in Figure 3.5. These transient errors are important for underresolved simulations. The use of the wave filter technology to identify the locations of the transient waves (when they have only marginally separated from one another) is a possible factor in our results, and we cannot clearly define the extent to which the transient position errors are an aspect of the simulation or of the diagnostic methods used to analyze it. In Figure 3.6 (left), ten of the cases out of the ensemble $(5 \%)$ did not produce usable wave filter data for early time transients. These cases were removed from the ensemble before averaging the ensemble for those time steps.

3.4. Shock crossing-shock interactions. Here we study the reflection of the shock off the wall, a special case of the shock crossing-shock interaction. We can ignore the shock wave width parameters, as these are narrow and deterministic. We consider only one output wave position error, $p_{1}^{o}$; see Table 3.2. The shock wave strength errors (the far field, large separation errors) are small and are not presented.

The contact mode contributes an error, well known as shock wall heating. The exact solution has no contribution in this mode for a wall reflection. The error is the imprint on the entropy, temperature, and density variables of entropy errors made during the shock interaction process, apparently due to shock oscillations. Since entropy can only increase, according to the second law of thermodynamics, these errors do not cancel. Because the solution algorithm conserves mass locally, we expect the spatial integral of the density errors to cancel approximately. Since the velocity of 

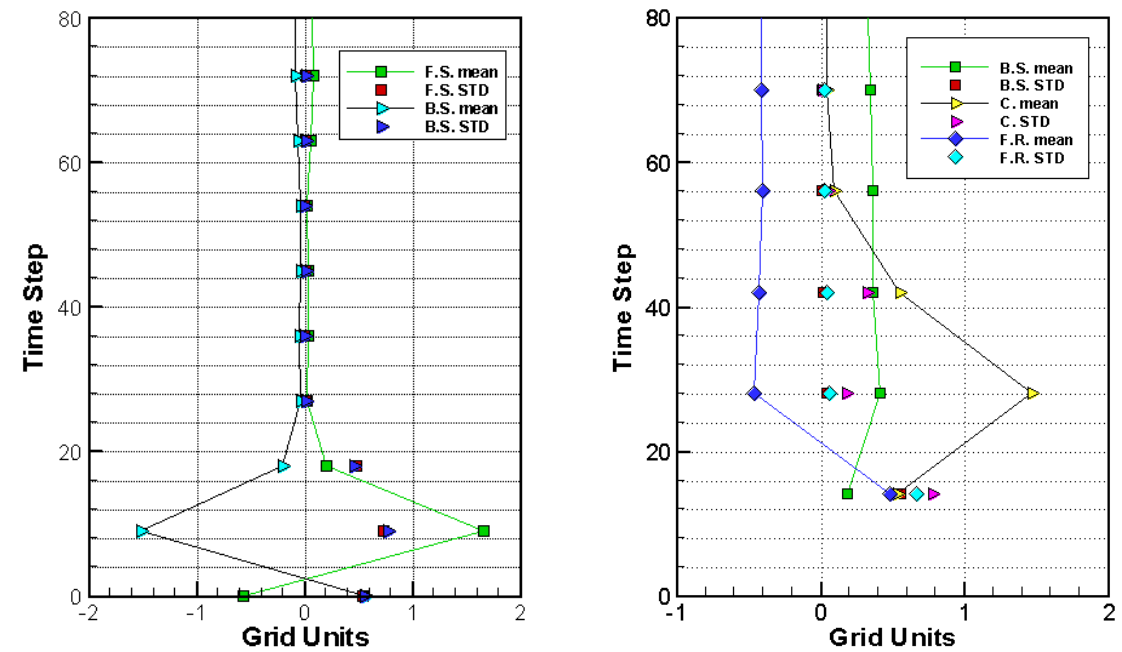

FIG. 3.6. Ensemble mean shock and contact position errors as a function of time, expressed in grid units. To emphasize the transient errors, a smaller number of time steps are shown. Left: shock crossing-shock case. Right: shock crossing-contact (step down) case.

TABLE 3.2

The SNRP defined by the crossing of two shocks. Expansion coefficients for output wave strengths (linear model) for input variation $\pm 10 \%$.

\begin{tabular}{|lrrrl|}
\hline Variable $\backslash$ Coef & Const & $\omega_{1}^{i}$ & \multicolumn{2}{c|}{ Error } \\
& & (r. sonic) & $L_{\infty}$ & STD \\
\hline$\omega_{1}^{o}$ (l. sonic) & -0.002 & 0.716 & $0.014 \%$ & 0.000032 \\
$\lambda_{1}^{o}$ (l. sonic) & 2.291 & -0.422 & $7.923 \%$ & 0.062 \\
$p_{1}^{o}$ (l. sonic) & 0.060 & -0.039 & $5065 \%$ & 0.009 \\
$\omega_{2}^{o}$ (contact) & 0.057 & 0.0003 & $24.4 \%$ & 0.005 \\
$\lambda_{2}^{o}$ (contact) & 5.9 & & $50 \%$ & 0.7 \\
\hline
\end{tabular}

the fluid at the wall is zero, these errors do not move, and they remain permanently attached to the wall. As a numerical wave, the error is a standing wave. We do not have a theoretical model for the form of these errors. Due to this lack, our fitting of the errors will be less precise than those discussed elsewhere in this paper. We define the wall error width to be the distance to the wall in mesh units of the furthest location for which the density error is at least twice the background noise in the postshock region, or about $1 \%$ of the base case density. This width is about $6 \Delta x$. Also the wave strength error is defined dimensionlessly as the $L_{1}$ error in density, divided by $\rho_{0} \Delta x$, where $\rho_{0}$ is the base case postshock density after the wall reflection.

3.5. The contact reshock interaction. After reflection from the wall, the transmitted lead shock wave recrosses the deflected contact. This is a step down interaction. We have one input and one output wave width parameter, both for the contact. According to the analysis of section 3.2, the contact width is modeled as $c_{c} t^{1 / 3}$ where both the width and $t$ are expressed in mesh units. This formula is accurate after some 50 time steps, according to Figure 3.4, and the Table 3.3 entry 
TABLE 3.3

The SNRP shock contact (step down) interaction. Expansion coefficients for output wave strengths (linear model) for input variation $\pm 10 \%$.

\begin{tabular}{|lrrrrr|}
\hline Variable $\backslash$ Coef & Const & $\omega_{1}^{i}$ & \multicolumn{2}{c}{$\omega_{2}^{i}$} & \multicolumn{2}{c|}{ Error } \\
& & (contact) & (l. sonic) & \multicolumn{1}{c}{$L_{\infty}$} & STD \\
\hline$\omega_{1}^{o}$ (l. sonic) & 0.282 & -0.314 & 0.645 & $0.57 \%$ & 0.0008 \\
$\omega_{2}^{o}$ (contact) & 0.013 & 0.819 & 0.118 & $0.20 \%$ & 0.0003 \\
$\omega_{3}^{o}$ (r. sonic) & -0.128 & 0.143 & 0.468 & $0.41 \%$ & 0.0004 \\
$\lambda_{1}^{o}$ (l. sonic) & 2.383 & 0.754 & -1.307 & $5.47 \%$ & 0.038 \\
$\lambda_{2}^{o}$ (contact) & 0.909 & 0.011 & 0.216 & $1.00 \%$ & 0.005 \\
$\lambda_{3}^{o}$ (r. sonic) & 3.619 & 0.151 & -0.974 & $14.8 \%$ & 0.138 \\
$p_{1}^{o}$ (l. sonic) & 0.242 & 0.043 & 0.042 & $10.4 \%$ & 0.014 \\
$p_{2}^{o}$ (contact) & -0.036 & 0.045 & 0.066 & $75.5 \%$ & 0.008 \\
$p_{3}^{o}$ (r. sonic) & -0.447 & 0.078 & -0.036 & $16.7 \%$ & 0.029 \\
\hline
\end{tabular}

$\lambda_{2}^{o}=c_{c}$ in this formula. We form a linear model for this constant in this expression in Table 3.3. The rarefaction width has the form constant + rate $\times$ time. We find very small errors in the rate, not tabulated here. The entry $\lambda_{3}^{o}$ refers to the constant, which gives an offset for the centering of the rarefaction wave. This entry is expressed in mesh units.

4. Composite shock interaction problems. Here we add up the pieces. We introduce a formula for combining the wave interaction errors defined in sections 2 and 3 for isolated Riemann problems to yield the error for arbitrary points in the solution of the complex wave interaction problem. The formula is validated for fully resolved simulations, and it is shown to be partially correct and partially incorrect for underresolved simulations.

4.1. A multipath integral for a nonlinear multiscattering problem. We begin with a formula expressing the error in a given Riemann problem $R_{0}$ as multinomial expansion associated with initial waves and errors located inside its domain of dependence. For a one-dimensional shock wave interaction problem, think of the solution as being primarily composed of localized waves, interacting through Riemann problems and generating outgoing waves, that further interact in the same manner. Each wave $w$ is described by a vector $\nu_{w}$ that records its strength, location in state space, speed and starting location and time, and the errors or uncertainty associated with these quantities. The interaction of waves generates a planar (one-dimensional space and time) graph, the vertices of which are the Riemann problems and the bonds are the traveling waves, between Riemann problem interactions. Starting from a given Riemann problem (vertex) or wave (bond), we can trace backward and determine its domain of dependence. Call this graph $\mathcal{G}$.

For each Riemann problem, we consider three types of vertices, corresponding to the constant, linear, and bilinear terms in the parameterized approximate solution and error terms developed in section 3 . We treat the linear terms separately from the others, as they allow a simple propagation law,

$$
\mathcal{S}_{L}=\int w(t=0) d \omega
$$

where $w(t=0)$ is a vector representing the strength of the time zero wave and its error or uncertainty, evaluated at the beginning of the path $\omega$, and $\mathcal{S}_{L}$ is the purely linear propagation contribution to a final time error. The path space integral $d \omega$ 


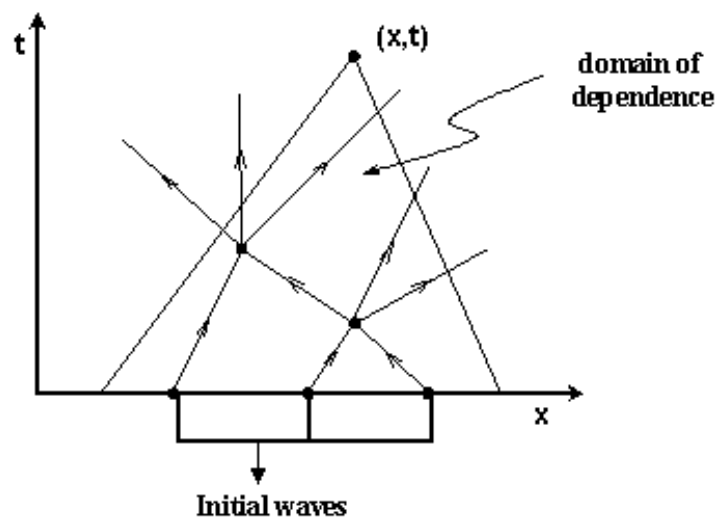

FIG. 4.1. The solution and its errors at the point $(x, t)$ can be obtained by "adding up" the solution and errors for the waves within the domain of dependence.

is taken over all paths progressing in time order through $\mathcal{G}$ from the initial time to the final vertex, with each term weighted by the appropriate linear factors from the formula for the approximate solution of the Riemann problems transversed. This path space representation makes evident the point that the solution $\mathcal{S}_{L}$ is that of a multiple (linear) scattering problem.

The amplitude $\mathcal{S}$ at the final time (vertex of $\mathcal{G}$ ) can similarly be thought of as a solution of a nonlinear multiple scattering problem, leading to a representation in terms of multipath integrals. To allow nonlinear (constant and bilinear) interactions, we reintroduce the vertices from these other terms. Let $\mathcal{V}=\mathcal{V}(\mathcal{G})$ be the set of vertices of $\mathcal{G}$, and let $\mathcal{B} \subset \mathcal{V}$ be a subset of $\mathcal{V}$ where constant or bilinear terms occur. The total amplitude $\mathcal{S}$ will then be a sum over terms $\mathcal{S}_{\mathcal{B}}$ indexed by $\mathcal{B}$. For each $v \in \mathcal{B}$, let $\mathcal{I}_{v}$ be the interaction coefficient, taken from a table of section 3 . We write

$$
\mathcal{S}=\sum_{\mathcal{B} \subset \mathcal{V}(\mathcal{G})} \mathcal{S}_{\mathcal{B}}=\sum_{\mathcal{B} \subset \mathcal{V}(\mathcal{G})} \int \prod_{v \in \mathcal{B}} \mathcal{I}_{v} d \omega_{\mathcal{B}}
$$

Here $d \omega_{\mathcal{B}}$ is a multipath integral over all multipaths (directed subgraphs of $\mathcal{G}$ starting at $t=0$ or at constant vertices, coalescing at bilinear vertices $v \in \mathcal{B}$, and ending at the final vertex in $\mathcal{G})$. The multipath propagator $d \omega_{\mathcal{B}}$ is a product of the individual propagators $\omega$ for each single path, as in (4.1). The summation in (4.2) can be understood schematically as the sum over all events within the domain of dependence of the evaluation point $(x, t)$ at the vertex of $\mathcal{G}$; see Figure 4.1.

4.2. Evaluation of the multipath integral. We consider, in one spatial dimension, the interaction of a shock wave with a contact located near a reflecting wall. The base case for the first wave interaction coincides with the base case for the shockcontact interaction studied in sections 2 and 3 . We further specify the wall location as 1.5 units to the right of the initial contact location. The transmitted shock, after interaction with the contact, progresses to interact with (i.e., reflect off) the wall. This interaction was also studied in sections 2 and 3. Subsequently, there are a number of reverberations, of reflected rarefactions and compression waves, between the contact and the wall and between a new contact formed by a shock overtake interaction and the original contact. The new contact is clearly visible in Figure 4.2 (left), as the 

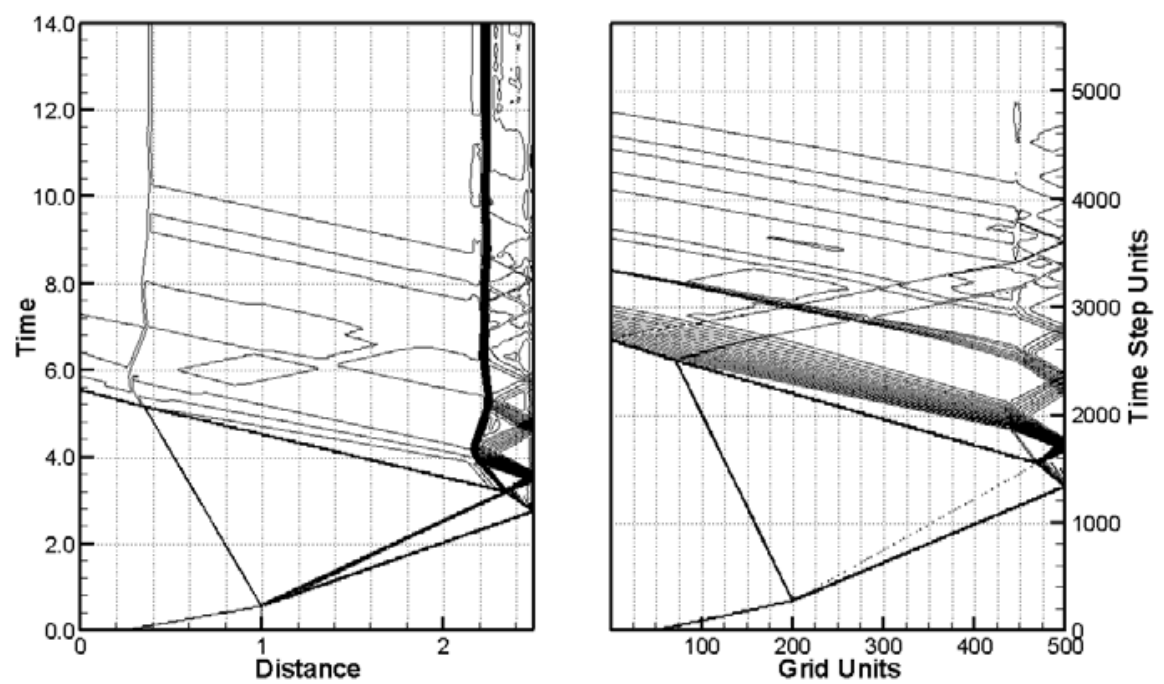

FIG. 4.2. Left: space-time density contour plots for the multiple wave interaction problem studied in this section. Right: pressure contour plots for the base case considered here.
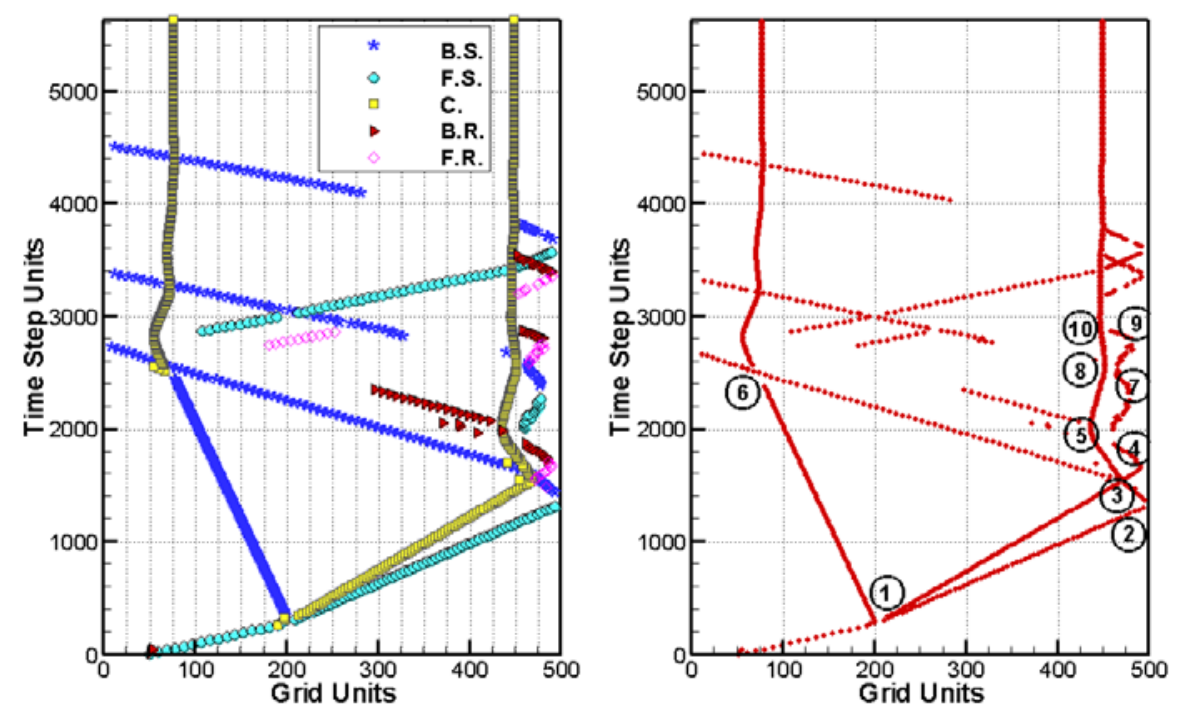

FIG. 4.3. Left: type and location of waves as determined by our wave filter analysis for the base case considered here. Right: schematic representation of the waves and the interactions, with labels for the interactions, taken from the left frame.

vertical line near the left border, starting at a time about $t=5.2$. The interactions are illustrated by the space-time contour plots of the density, shown in Figure 4.2 (left) and pressure contours (right). In Figure 4.3 (left), we show the type and location of the waves, as determined by our wave filter analysis program. Both figures refer to the base case. The buildup of complex wave patterns is evident. 

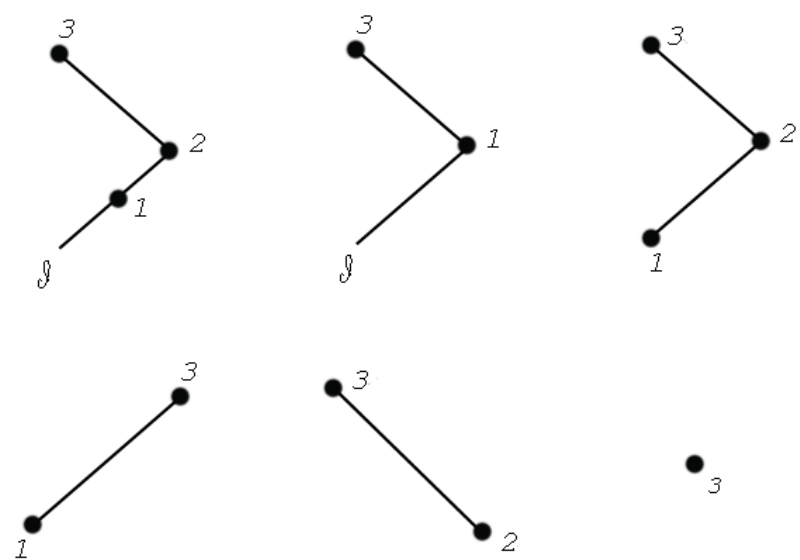

FIG. 4.4. Schematic graphs illustrating all contributions to the errors or uncertainty in the output from a single Riemann solution, namely the reshock interaction (case 3) of the reflected shock from the wall as it crosses the contact. The numbers labeling the black circles refer to the Riemann interactions contributing to the error. The letter $\mathcal{I}$ in the first two diagrams indicates input uncertainty.

Ten Riemann problems are extracted from the complex wave problem interaction illustrated in Figure 4.3 (left). A schematic representation of the wave interactions, identifying these ten Riemann problems is given in Figure 4.3 (right). In Figure 4.4 we illustrate the distinct terms contributing to (4.2). Each graph is a single term, for the error associated with the output to interaction 3 , in which the shock wave reflected from the wall recrosses (reshocks) the contact. The first two graphs indicate the uncertainty originating with the initial conditions, i.e., with the choice of the ensemble. This uncertainty propagates through two distinct paths, illustrated by the first two graphs of Figure 4.4, to reach the interaction site 3. The first graph follows the shocks, the transmitted shock from the interaction 1 to the wall reflected shock and back to the contact. The second graph follows the contact from the lead interaction 1 along the contact until it is reshocked at interaction 3. Next we find two graphs that represent the errors originating during the interaction 1 and propagating to the output of 3 through the same two routes. Finally, we find two graphs giving the errors that arise at the shock wall reflection (interaction 2) and propagate to 3 and in the final graph, those arising during interaction 3 directly.

From prior work [6], we know that the dominant errors in the composite solution are located within the leading shock and contact waves of the problem. A portion of these errors are simple resolution errors. This means that they are errors due to the difference between the numerical and the exact wave forms as traveling waves. This portion of the error is independent of the wave interactions and, in the variables we use to describe the traveling waves, shows up in the wave width only. Any other errors, e.g., in strength or position, or errors in width beyond these resolution errors can be attributed to the wave interactions.

Now we explain in detail the definition of each of the terms associated with these graphs. The simplest is the last. It is the error created during the solution of the interaction at Riemann problem case 3 . For this term, we accept the numerical input to case 3 (as defined by a coarse grid solution, including the numerical error). This 
input data is solved using both a fine grid and a coarse grid, and the difference is the error associated with this graph. The same formula applied to the initial point of the other graphs defines the beginning of each of the graphs 3-5. From this input error, we proceed as follows: The error is transmitted without change along the edges of the graph. For graph 3, the error is transmitted from output of the first interaction of the graph, that is, the pure transmitted shock wave output of interaction case 1 to the input of interaction case 2 in the same graph. The case 3 Riemann problem then sees this error as an initial uncertainty and transforms it via a linear transformation to an output error or uncertainty located at the output of interaction 3. The definition of the other graphs is similar, containing a sequence of linear transformations, one for each Riemann problem the error signal passes through.

We develop these formulas explicitly for the first two graphs of Figure 4.4, representing one component of the propagation of the initial uncertainty (initial ensemble) to the output uncertainty of interaction 3. The initial uncertainty is reflected in the wave strength variables according to the definition of the ensemble of initial conditions. The transmission of the mean values through a linear model is standard and is not detailed here. The transformation of the variance follows formulas from section 2 . Let $B^{(l)}$ with matrix entries $\beta_{j k}^{(l)}$ be the matrix that gives the linear transformation of these variables due to interaction $l$, as in (2.10). We note that the matrix entries $\beta_{j k}^{(l)}$ are defined by the $\omega^{i}$ columns and $\omega^{o}$ rows of Table 2.1 (linear; $l=1$ ), Table 2.3 $(l=2)$, and Table $6.3(l=3)$. The output to interaction 3 has three components, and we compute the variance of each, labeled $j=1,2,3$. By formula (2.13), we have

$$
\begin{aligned}
\operatorname{Var} \omega_{j}^{o(3)} & =\left(\beta_{j 2}^{(3)}\right)^{2} \operatorname{Var} \omega_{2}^{i(3)}=\left(\beta_{j 2}^{(3)}\right)^{2} \operatorname{Var} \omega_{1}^{o(2)} \\
& =\left(\beta_{j 2}^{(3)}\right)^{2}\left(\beta_{11}^{(2)}\right)^{2} \operatorname{Var} \omega_{1}^{i(2)}=\left(\beta_{j 2}^{(3)}\right)^{2}\left(\beta_{11}^{(2)}\right)^{2} \operatorname{Var} \omega_{3}^{o(1)} \\
& =\left(\beta_{j 2}^{(3)}\right)^{2}\left(\beta_{11}^{(2)}\right)^{2} \sum_{k=1}^{2} \operatorname{Var}\left(\beta_{3 k}^{(1)}\right)^{2} \omega_{k}^{i(1)} .
\end{aligned}
$$

This formula can be evaluated explicitly. We denote the left-hand side as $\mathcal{S}_{\mathcal{B}_{1}}$ in accordance with (4.2):

$$
\begin{aligned}
\mathcal{S}_{\mathcal{B}_{1}} & =\left(\begin{array}{ll}
0.312^{2} & 0.645^{2} \\
0.809^{2} & 0.124^{2} \\
0.142^{2} & 0.468^{2}
\end{array}\right)\left(\begin{array}{c}
0 \\
\operatorname{Var}\left(w_{2}^{i_{3}}\right)
\end{array}\right) \\
(4.4) & =\left(\begin{array}{ll}
0.312^{2} & 0.645^{2} \\
0.809^{2} & 0.124^{2} \\
0.142^{2} & 0.468^{2}
\end{array}\right) 0.715^{2}\left(\begin{array}{cc}
0 & 0 \\
1.001^{2} & 0.347^{2}
\end{array}\right)\left(\begin{array}{c}
\operatorname{Var}\left(w_{1}^{i}(t=0)\right) \\
\operatorname{Var}\left(w_{2}^{i}(t=0)\right)
\end{array}\right) .
\end{aligned}
$$

Similarly, the variance associated with the second graph can be evaluated as

$$
\begin{aligned}
\mathcal{S}_{\mathcal{B}_{2}} & =\left(\begin{array}{ll}
0.312^{2} & 0.645^{2} \\
0.809^{2} & 0.124^{2} \\
0.142^{2} & 0.468^{2}
\end{array}\right)\left(\begin{array}{cc}
\operatorname{Var}\left(w_{1}^{i_{3}}\right) \\
0
\end{array}\right) \\
& =\left(\begin{array}{ll}
0.312^{2} & 0.645^{2} \\
0.809^{2} & 0.124^{2} \\
0.142^{2} & 0.468^{2}
\end{array}\right)\left(\begin{array}{cc}
0.000^{2} & 0.911^{2} \\
0 & 0
\end{array}\right)\left(\begin{array}{c}
\operatorname{Var}\left(w_{1}^{i}(t=0)\right) \\
\operatorname{Var}\left(w_{2}^{i}(t=0)\right)
\end{array}\right) .
\end{aligned}
$$

We also need to calculate formulas giving the transmission of position errors through the various Riemann problems. For interaction with a wall (e.g., case 2), the 
formula is elementary. Assuming no error in the wall position, let $p^{i}$ and $p^{o}$ denote input and output position errors for a reflection off of a stationary wall, where the output is due transmission of error, i.e., due only to the input, as opposed to section 3 , where there is no input position error and the output position error is created during the interaction. Then we have

$$
p^{o}=p^{i} \frac{v^{o}}{v^{i}},
$$

where $v^{i}$ and $v^{o}$ are the incoming and outgoing wave speeds for the waves involved in the wall reflection. For the interaction of two incoming waves, the result is slightly more complicated. Each of the two terms in the formulas below is due to the input error in one of the input waves. That error can be computed by (4.6) if we perform the analysis in the frame in which the other wave is stationary. The result is

$$
\begin{aligned}
& p_{1}^{o}=\frac{p_{1}^{i}\left(v_{1}^{o}-v_{2}^{i}\right)+p_{2}^{i}\left(v_{1}^{i}-v_{1}^{o}\right)}{v_{1}^{i}-v_{2}^{i}}, \\
& p_{2}^{o}=\frac{p_{1}^{i}\left(v_{2}^{o}-v_{2}^{i}\right)+p_{2}^{i}\left(v_{1}^{i}-v_{2}^{o}\right)}{v_{1}^{i}-v_{2}^{i}}, \\
& p_{3}^{o}=\frac{p_{1}^{i}\left(v_{3}^{o}-v_{2}^{i}\right)+p_{2}^{i}\left(v_{1}^{i}-v_{3}^{o}\right)}{v_{1}^{i}-v_{2}^{i}}
\end{aligned}
$$

where $p_{j}^{i}$ is the position error of the incoming contact wave $(j=1)$ or left-facing shock $(j=2)$. The complete position error model is obtained by adding the results of (4.7), (4.8), and (4.9) to those of Table 3.3 for the position errors created at the interactions. For interactions 1-3, we model the wave width (error) as a created error only. Thus only the final graph of Figure 4.4 contributes to this.

4.3. Errors in resolved calculations. We regard a calculation as resolved if all (the principal) waves have separated, with converged left and right asymptotic states, before they interact with another wave. For this type of simulation, we choose 500 mesh cells in our basic simulation study and assess errors in comparison to a 5000-cell simulation. We examine errors in wave strength, wave position, and wave width, based on the graphical expansion given in sections 4.1 and 4.2. The wave strength errors are dominated by the transmission of error (or uncertainty) from the initial conditions, and thus are given by the first two diagrams of Figure 4.4. In Tables $4.1,4.2$, and 4.3 we compare the predicted error with the error computed directly, taken from a full solution of the multiple wave interaction problem. The model for the prediction of the error is satisfactory for all cases: the wave strength and its errors, the wave width errors, and the wave position errors.

4.4. Errors in underresolved calculations. Here we allow 100 cells for the coarse grid simulation. This resolution allows 10 cells between the contact and the reflection wall at the time of interaction 3 and beyond. Since the contact has a width of 5 cells, since the right facing rarefaction is about this size, and since the wall has inaccurate states in a region of several mesh blocks neighboring it, we are clearly at the limit of the present diagnostic methods based upon the wave filter. For the same reasons, the calculation is clearly underresolved. For this reason we are not able to analyze data for the case of a coarse grid simulation with 50 cells using the present 
TABLE 4.1

Predicted and simulated errors for output wave strengths, wave widths, and wave positions; Case 1.

\begin{tabular}{|lcc|}
\hline Variable $\backslash$ Error & Simulation & Prediction \\
\hline \multicolumn{3}{|c|}{ Mean wave strengths } \\
$\omega_{1}^{o}$ (l. sonic) & 0.451 & 0.452 \\
$\omega_{2}^{o}$ (contact) & 0.704 & 0.703 \\
$\omega_{3}^{o}$ (r. sonic) & 0.999 & 0.998 \\
\hline \multicolumn{3}{|c|}{ Wave strength errors } \\
$\operatorname{Var} \omega_{1}^{o}$ (l. sonic) & 0.0008 & 0.0008 \\
$\operatorname{Var} \omega_{2}^{o}$ (contact) & 0.0019 & 0.0018 \\
$\operatorname{Var} \omega_{3}^{o}$ (r. sonic) & 0.0035 & 0.0036 \\
\hline \multicolumn{3}{|c}{ Wave width errors } \\
$\lambda_{1}^{o}$ (l. sonic) & 1.630 & 1.622 \\
$\lambda_{2}^{o}$ (contact) & 3.636 & 3.635 \\
$\lambda_{3}^{o}$ (r. sonic) & 2.346 & 2.352 \\
\hline \multicolumn{3}{|c}{ Wave position errors } \\
$p_{1}^{o}$ (l. sonic) & 0.220 & 0.226 \\
$p_{2}^{o}$ (contact) & 0.313 & 0.312 \\
$p_{3}^{o}$ (r. sonic) & 0.200 & 0.202 \\
\hline
\end{tabular}

TABLE 4.2

Predicted and simulated errors for output wave strengths, wave widths, and wave positions; Case 2.

\begin{tabular}{|ccc|}
\hline Variable $\backslash$ Error & Simulation & Prediction \\
\hline \multicolumn{4}{|c|}{ Mean wave strengths } \\
$\omega_{1}^{o}$ (l. sonic) & 0.713 & 0.714 \\
\hline \multicolumn{4}{|c|}{ Wave strength errors } \\
Var $\omega_{1}^{o}$ (l. sonic) & 0.0018 & 0.0018 \\
\hline \multicolumn{4}{|c}{ Wave width errors } \\
$\lambda_{1}^{o}$ (l. sonic) & 1.868 & 1.869 \\
\hline \multicolumn{4}{|c}{ Wave position errors } \\
$p_{1}^{o}$ (l. sonic) & -0.118 & -0.092 \\
\hline
\end{tabular}

TABle 4.3

Predicted and simulated errors for output wave strengths, wave widths, and wave positions; Case 3 .

\begin{tabular}{|ccc|}
\hline Variable $\backslash$ Error & Simulation & Prediction \\
\hline \multicolumn{3}{|c|}{ Mean wave strengths } \\
$\omega_{1}^{o}$ (l. sonic) & 0.520 & 0.519 \\
$\omega_{2}^{o}$ (contact) & 0.674 & 0.674 \\
$\omega_{3}^{o}$ (r. sonic) & 0.306 & 0.305 \\
\hline \multicolumn{4}{|c|}{ Wave strength errors } \\
$\operatorname{Var} \omega_{1}^{o}$ (l. sonic) & 0.0009 & 0.0010 \\
$\operatorname{Var} \omega_{2}^{o}$ (contact) & 0.0012 & 0.0013 \\
$\operatorname{Var} \omega_{3}^{o}$ (r. sonic) & 0.0004 & 0.0004 \\
\hline \multicolumn{4}{|c}{ Wave width errors } \\
$\lambda_{1}^{o}$ (l. sonic) & 2.097 & 1.982 \\
$\lambda_{2}^{o}$ (contact) & 5.027 & 4.918 \\
$\lambda_{3}^{o}$ (r. sonic) & 2.875 & 3.033 \\
\hline \multicolumn{4}{c}{ Wave position errors } \\
$p_{1}^{o}$ (l. sonic) & -0.097 & -0.105 \\
$p_{2}^{o}$ (contact) & -0.003 & 0.013 \\
$p_{3}^{o}$ (r. sonic) & -0.151 & -0.134 \\
\hline \multicolumn{4}{c}{} \\
\hline
\end{tabular}


TABLE 4.4

Case 1. The contact-shock interaction (step up). Errors for output wave strengths, wave widths, and wave position. Comparison of underresolved simulation and prediction.

\begin{tabular}{|ccc|}
\hline Variable $\backslash$ Error & Simulation & Prediction \\
\hline \multicolumn{3}{|c|}{ Mean wave strengths } \\
$\omega_{1}^{o}$ (l. sonic) & 0.451 & 0.452 \\
$\omega_{2}^{o}$ (contact) & 0.741 & 0.703 \\
$\omega_{3}^{o}$ (r. sonic) & 0.996 & 0.998 \\
\hline \multicolumn{4}{|c|}{ Wave strength errors } \\
$\operatorname{Var} \omega_{1}^{o}$ (l. sonic) & 0.0008 & 0.0008 \\
$\operatorname{Var} \omega_{2}^{o}$ (contact) & 0.0022 & 0.0018 \\
$\operatorname{Var} \omega_{3}^{o}$ (r. sonic) & 0.0036 & 0.0036 \\
\hline \multicolumn{4}{|c}{ Wave width errors } \\
$\lambda_{1}^{o}$ (l. sonic) & 1.381 & 1.621 \\
$\lambda_{2}^{o}$ (contact) & 3.498 & 3.635 \\
$\lambda_{3}^{o}$ (r. sonic) & 2.347 & 2.352 \\
\hline \multicolumn{4}{c}{ Wave position errors } \\
$p_{1}^{o}$ (l. sonic) & 0.972 & 0.226 \\
$p_{2}^{o}$ (contact) & 1.539 & 0.312 \\
$p_{3}^{o}$ (r. sonic) & 0.785 & 0.202 \\
\hline
\end{tabular}

TABLE 4.5

Case 2. The shock crossing-equal shock (wave reflection) interaction. Errors for output wave strengths, wave width, and wave position. Comparison of underresolved simulation and prediction.

\begin{tabular}{|ccc|}
\hline Variable $\backslash$ Error & Simulation & Prediction \\
\hline \multicolumn{4}{|c|}{ Mean wave strengths } \\
$\omega_{1}^{o}$ (l. sonic) & 0.721 & 0.712 \\
\hline \multicolumn{4}{|c}{ Wave strength errors } \\
Var $\omega_{1}^{o}$ (l. sonic) & 0.0018 & 0.0018 \\
\hline \multicolumn{4}{|c}{ Wave width errors } \\
$\lambda_{1}^{o}$ (l. sonic) & 1.718 & 1.871 \\
\hline \multicolumn{4}{|c|}{ Wave position errors } \\
$p_{1}^{o}$ (l. sonic) & -0.401 & -0.092 \\
\hline
\end{tabular}

version of our wave filter. Again we present the first three interactions in detail, at 100cell resolution, comparing the predicted to the directly simulated errors; see Tables 4.4, 4.5, and 4.6. We see good results for the wave strengths and their errors and for the wave width errors, and poor results for the comparison of position errors. This can be understood in terms of the decay time for convergence to asymptotic large time values for the position errors, an explanation that also accounts for the difference with the resolved case, for which the simulated and predicted position errors agree. The position errors have a relatively slower decay time. The other three quantities show a high level of agreement between the resolved and underresolved cases. The wave width error is expressed in grid units, and thus should be the same in the two cases differing in grid resolution only. For the wave strength entries, the lack of dependence on grid resolution is due to the fact that these quantities are dominated by the uncertainty expressed in the ensemble of initial conditions, which is independent of grid resolution.

5. Conclusions. We have several main conclusions from this study.

We see that a very simple model of solution error is sufficient for the study of (at least the present instance of) a highly nonlinear problem. The error is linear in the input wave strengths. 
TABLE 4.6

Case 3. The contact-shock interaction (step down). Errors for output wave strengths, wave width, and wave position. Comparison of underresolved simulation and prediction.

\begin{tabular}{|ccc|}
\hline Variable $\backslash$ Error & Simulation & Prediction \\
\hline \multicolumn{3}{|c|}{ Mean wave strengths } \\
$\omega_{1}^{o}$ (l. sonic) & 0.523 & 0.514 \\
$\omega_{2}^{o}$ (contact) & 0.669 & 0.705 \\
$\omega_{3}^{o}$ (r. sonic) & 0.318 & 0.315 \\
\hline \multicolumn{3}{|c|}{ Wave strength errors } \\
$\operatorname{Var} \omega_{1}^{o}$ (l. sonic) & 0.0009 & 0.0010 \\
$\operatorname{Var} \omega_{2}^{o}$ (contact) & 0.0013 & 0.0013 \\
$\operatorname{Var} \omega_{3}^{o}$ (r. sonic) & 0.0005 & 0.0004 \\
\hline \multicolumn{4}{|c}{ Wave width errors } \\
$\lambda_{1}^{o}$ (l. sonic) & 1.964 & 2.000 \\
$\lambda_{2}^{o}$ (contact) & 4.606 & 4.928 \\
$\lambda_{3}^{o}$ (r. sonic) & 2.169 & 3.029 \\
\hline \multicolumn{4}{c}{ Wave position errors } \\
$p_{1}^{o}$ (l. sonic) & -0.551 & -0.103 \\
$p_{2}^{o}$ (contact) & 0.301 & 0.015 \\
$p_{3}^{o}$ (r. sonic) & 0.432 & -0.131 \\
\hline
\end{tabular}

A composition law for combining errors and predicting errors for composite interactions on the basis of an error model of the simple constituent interactions has been formulated and validated.

We find, although our formalism allows for statistical errors in the ensemble, that in fact, the dominant part of all errors (excluding position errors) studied were deterministic, in the sense that the ensemble mean error dominated the ensemble standard deviation.

We find that the wave filter performs well as a diagnostic tool but that its limitation (in its present version) lies in assuming well-separated waves. Thus we are limited in the degree of underresolution that we can analyze, in that all waves must be at least partially separated from one another before entering into a new interaction.

The wave strength uncertainty, which is dominated by input uncertainty (i.e., the definition of the ensemble), is virtually unchanged between the highly resolved and the underresolved simulations. The wave width errors are both expressed in grid units and are comparable between the two levels of resolution. The wave width errors evidentially have a rapid relaxation to their asymptotic value.

The primary solution errors created by the simulations are the wave position errors in the underresolved simulations on the order of a mesh spacing. These errors are a transient phenomena but become frozen into the calculation as new interactions occur before the transient errors have diminished. The wave position errors have a slow relaxation to asymptotic values.

To the extent that a more detailed modelling of these errors is important, a more accurate model that includes transient effects will be important. Even with these limitations, the methods and results appear to be promising, and should be extended to less idealized problems.

These conclusions are established only under several simplifying assumptions, namely restriction to one spatial dimension, use of a simplified (gamma law gas) equation of state, and consideration of only one numerical method. Further studies are needed to determine the extent that these conclusions have a general validity. 
6. Appendix: Ten Riemann problems. For each of the ten Riemann problems of Figure 4.3 (right), we vary the wave strength and, for contacts only, we vary the wave width. However, when solved using the (idealized) Riemann solver, the wave widths are all set to zero. Three variables defining one reference state are not varied in this study; presumably similar conclusions would be reached if they were also varied. We have two goals in selecting the reference variables to hold fixed. If one of the states has a reference ambient velocity, for example, a velocity $v=0$ for a state near a wall, we want to preserve this property and freeze this velocity. For the pressure and density values, we generally freeze those on the smaller side of the waves, as this gives a more meaningful variation of the state, uniformly specified as $10 \%$ of the wave strength, as defined in section 2 .

6.1. Case 1: Lead shock interacts with contact. The midstate is held fixed, and the two wave strengths are varied.

$$
\begin{array}{l|ll|l}
\begin{array}{l}
\rho=3.973980 \\
p=1.337250 \\
v=1.0
\end{array} & \begin{array}{l}
\rho=1.0 \\
p=0.001 \\
v=0.0
\end{array} & \begin{array}{l}
\rho=10.0 \\
p=0.001 \\
v=0.0
\end{array} \\
\text { shock (width=0) } & \text { contact (width=0) }
\end{array}
$$

FIG. 6.1. Problem 1: Shock-contact interaction (step up).

TABLE 6.1

Case 1. The contact-shock interaction (step up). Expansion coefficients for output wave

\begin{tabular}{|c|c|c|c|c|c|}
\hline \multirow[t]{2}{*}{ Variable \Coef } & \multirow[t]{2}{*}{ Const } & \multirow{2}{*}{$\begin{array}{l}w_{1}^{i} \\
\text { (r. sonic) }\end{array}$} & \multirow{2}{*}{$\begin{array}{l}w_{2}^{i} \\
\text { (contact) }\end{array}$} & \multicolumn{2}{|c|}{ Error } \\
\hline & & & & $L_{\infty}$ & STD \\
\hline & \multicolumn{5}{|c|}{$10 \%$ input variation } \\
\hline$w_{1}^{o}(1$ & -0.206 & 0.452 & 0.252 & $0.55 \%$ & 0.0008 \\
\hline$w_{2}^{o}($ contact $)$ & -0.042 & 0.000 & 0.911 & $0.01 \%$ & 0.00004 \\
\hline$w_{3}^{o}$ (r. sonic) & -0.285 & 1.001 & 0.347 & $0.35 \%$ & 0.001 \\
\hline
\end{tabular}
strengths (linear model). See Tables 2.1 and 2.2.

6.2. Case 2: Transmitted shock reflects off of wall. The right state is held fixed, and the left state is varied.

$$
\begin{aligned}
& \rho=39.808728 \\
& p=3.537544 \\
& v=0.514603
\end{aligned}
$$

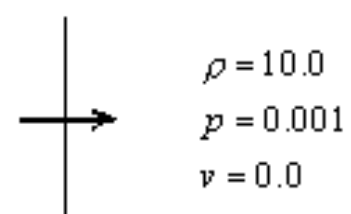

shock (width=2.3)

FIG. 6.2. Problem 2: Shock-wall interaction. 
TABLE 6.2

Case 2. The shock crossing-equal shock (wall reflection) interaction. Expansion coefficients for output wave strengths (linear model) for input variation $\pm 10 \%$. See Table 2.3 .

\begin{tabular}{|c|c|c|c|c|}
\hline \multirow[t]{2}{*}{ Variable $\backslash$ Coef } & \multirow[t]{2}{*}{ Const } & \multirow{2}{*}{$\begin{array}{l}\quad w_{1}^{i} \\
\text { (r. sonic) }\end{array}$} & \multicolumn{2}{|c|}{ Error } \\
\hline & & & $L_{\infty}$ & STD \\
\hline & \multicolumn{4}{|c|}{$10 \%$ input variation } \\
\hline$w_{1}^{o}$ (l. sonic) & -0.0008 & 0.715 & $0.00 \%$ & $4 \mathrm{E}-8$ \\
\hline
\end{tabular}

6.3. Case 3: Shock reflected off of wall recrosses the contact. The right, state velocity $v=0.0$ is fixed, and the left state densities and pressures are held fixed.

$$
\begin{array}{l|l|l}
\rho=6.917758 & \rho=39.808728 & \\
p=3.537544 & \begin{array}{l}
\rho=99.182916 \\
p=3.537544 \\
v=0.514603
\end{array} & \begin{array}{l}
p=21.147657 \\
v=0.0
\end{array}
\end{array}
$$

contact (width=3.8) shock (width=1.9)

Fig. 6.3. Problem 3: Contact-shock interaction (step down).

TABLE 6.3

Case 3. The contact-shock interaction (step down). Expansion coefficients for output wave strengths (linear model).

\begin{tabular}{|c|c|c|c|c|c|}
\hline \multirow{2}{*}{ Variable $\backslash$ Coef } & \multirow[t]{2}{*}{ Const } & \multirow{2}{*}{$\begin{array}{r}w_{1}^{i} \\
\text { (contact) }\end{array}$} & \multirow{2}{*}{$\begin{array}{l}w_{2}^{i} \\
\text { (l. sonic) }\end{array}$} & \multicolumn{2}{|c|}{ Error } \\
\hline & & & & $L_{\infty}$ & STD \\
\hline & \multicolumn{5}{|c|}{$10 \%$ input variation } \\
\hline$w_{1}^{o}$ (l. sonic) & 0.281 & -0.314 & 0.646 & $0.62 \%$ & 0.0008 \\
\hline$w_{2}^{o}$ (contact) & 0.016 & 0.810 & 0.124 & $0.15 \%$ & 0.0003 \\
\hline$w_{3}^{o}(\mathrm{r}$. sonic $)$ & -0.128 & 0.143 & 0.468 & $0.48 \%$ & 0.0006 \\
\hline
\end{tabular}

6.4. Case 4: Reflected rarefaction from Case 3 reflects off of wall. The right state velocity $v=0.0$ is fixed, and the left state densities and pressures are held fixed. When modeled as an SRP, the input rarefaction wave width is set to zero. When modeled as an SNRP, the input rarefaction wave width is an input parameter. Similar comments apply to the most of the later cases.

$$
\begin{array}{l|l}
\rho=67.888252 \\
p=11.228189 \\
v=-0.212443
\end{array} \longrightarrow \begin{aligned}
& \rho=99.182916 \\
& p=21.147657 \\
& v=0.0
\end{aligned}
$$

raref action (width $=13.7$ )

FIG. 6.4. Problem 4: Rarefaction-wall interaction. 
TABLE 6.4

Case 4. The rarefaction-wall interaction. Expansion coefficients for output wave strengths (linear model).

\begin{tabular}{|lllll|}
\hline Variable $\backslash$ Coef & Const & \multicolumn{2}{c|}{$w_{1}^{i}$} & \multicolumn{2}{c|}{ Error } \\
& & (r. sonic) & $L_{\infty}$ & STD \\
\hline$w_{1}^{o}$ (l. sonic) & 0.147 & 0.656 & $0.189 \%$ & 0.0002 \\
\hline
\end{tabular}

6.5. Case 5: Rarefaction reflected off of wall crosses contact. The right state velocity $v=0.0$ is fixed, and the left state densities and pressures are held fixed.

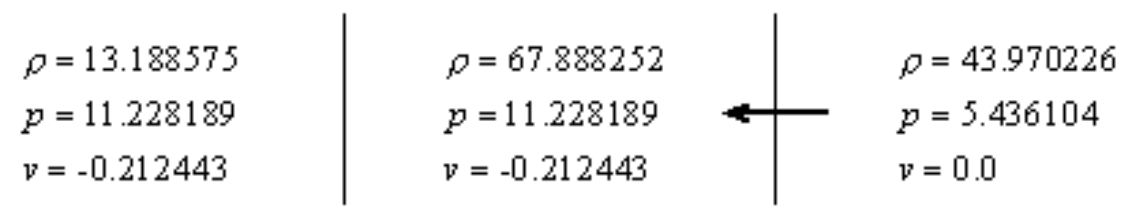

contact (width $=7.3) \quad$ rarefaction $($ width $=3.4)$

FIG. 6.5. Problem 5: Contact-rarefaction interaction.

TABLE 6.5

Case 5. The contact-rarefaction interaction (step down). Expansion coefficients for output wave strengths (linear model).

\begin{tabular}{|lrrrrr|}
\hline Variable $\backslash$ Coef & Const & $w_{1}^{i}$ & $w_{2}^{i}$ & \multicolumn{2}{c|}{ Error } \\
& & (contact) & (l. sonic) & $L_{\infty}$ & STD \\
\hline & & $10 \%$ & input variation \\
$w_{1}^{o}$ (l. sonic) & 0.020 & -0.074 & 0.698 & $0.37 \%$ & 0.0001 \\
$w_{2}^{o}$ (contact) & 0.200 & 1.072 & -0.721 & $0.35 \%$ & 0.0007 \\
$w_{3}^{o}$ (r. sonic) & -0.029 & 0.107 & 0.294 & $0.71 \%$ & 0.0003 \\
\hline
\end{tabular}

6.6. Case 6: Reflected shocks from interactions 1 and 3 overtake. The left state is held fixed, and the two wave strengths are varied.

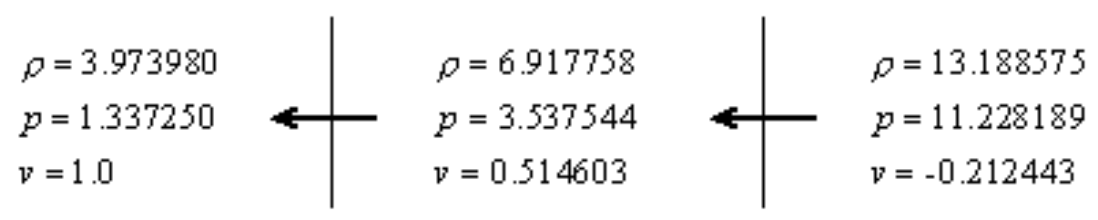

shock (width $=1.32)$

shock (width $=2.1)$

FiG. 6.6. Problem 6: Shock-shock overtake (two waves of the same family). 
TABLE 6.6

Case 6. The shock-shock overtake (two waves of the same family). Expansion coefficients for output wave strengths (linear model).

\begin{tabular}{|crlllll|}
\hline Variable $\backslash$ Coef & Const & $w_{1}^{i}$ & $w_{2}^{i}$ & \multicolumn{2}{c|}{ Error } \\
& & (l.sonic) & (l. sonic) & $L_{\infty}$ & STD \\
\hline & \multicolumn{4}{c}{$10 \%$ input variation } \\
$w_{1}^{o}$ (l. sonic) & 0.033 & 0.375 & 1.110 & $0.02 \%$ & 0.0001 \\
$w_{2}^{o}$ (m. sonic) & -0.029 & 0.075 & 0.150 & $0.88 \%$ & 0.0002 \\
$w_{3}^{o}$ (r. sonic) & -0.018 & 0.014 & 0.055 & $0.61 \%$ & 0.0001 \\
\hline
\end{tabular}

6.7. Case 7: Compression wave reflected from interaction 5 reflects off of wall. The right state is held fixed.

$$
\begin{array}{l|l}
\rho=52.397949 \\
p=7.295522 \\
v=0.082472
\end{array} \longrightarrow \begin{aligned}
& \rho \\
& p=43.970226 \\
& v=0.0
\end{aligned}
$$

FIG. 6.7. Problem 7: Compression-wall interaction.

TABLE 6.7

Case 7. The compression-wall interaction. Expansion coefficients for output wave strengths (linear model).

\begin{tabular}{|lllll|}
\hline Variable $\backslash$ Coef & Const & $w_{1}^{i}$ & \multicolumn{2}{c|}{ Error } \\
& & (r. sonic) & $L_{\infty}$ & STD \\
\hline$w_{1}^{o}$ (l. sonic) & -0.026 & 1.125 & $0.14 \%$ & 0.0001 \\
\hline
\end{tabular}

6.8. Case 8: Compression wave from wall reflection crosses contact. The right state velocity $v=0.0$ is fixed, and the left state densities and pressures are held fixed.

$$
\begin{aligned}
& \rho=10.187558 \\
& \rho=52.397949 \\
& p=7.295522 \\
& p=7.295522 \\
& v=0.082472 \\
& v=0.082472 \\
& \rho=61.835041 \\
& p=9.630720 \\
& v=0.0
\end{aligned}
$$

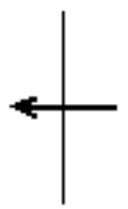

FIG. 6.8. Problem 8: Contact-compression interaction. 
TABLE 6.8

Case 8. The contact-compression interaction (step down). Expansion coefficients for output wave strengths (linear model).

\begin{tabular}{|c|c|c|c|c|c|}
\hline \multirow{2}{*}{ Variable \Coef } & \multirow[t]{2}{*}{ Const } & \multirow{2}{*}{$\begin{array}{r}w_{1}^{i} \\
(\text { contact) }\end{array}$} & \multirow{2}{*}{$\begin{array}{l}w_{2}^{i} \\
\text { (l. sonic) }\end{array}$} & \multicolumn{2}{|c|}{ Error } \\
\hline & & & & $L_{\infty}$ & STD \\
\hline & \multicolumn{5}{|c|}{$10 \%$ input variation } \\
\hline$w_{1}^{o}($ l. sonic $)$ & 0.029 & -0.038 & 0.598 & $0.37 \%$ & 0.0001 \\
\hline$w_{2}^{o}($ contact $)$ & -0.041 & 0.971 & 0.432 & $0.07 \%$ & 0.0001 \\
\hline$w_{3}^{o}$ (r. sonic) & -0.025 & 0.033 & 0.404 & $0.00 \%$ & 0.0001 \\
\hline
\end{tabular}

6.9. Case 9: Rarefaction reflected from interaction 8 reflects off of wall. The right state velocity $v=0.0$ is fixed, and the left state densities and pressures are held fixed.

$$
\begin{aligned}
& \rho=58.035866 \\
& p=8.663034 \\
& v=-0.031998
\end{aligned} \longrightarrow \longrightarrow \begin{aligned}
& \rho=61.835041 \\
& p=9.630720 \\
& v=0.0
\end{aligned}
$$

rarefaction (width $=28.0$ )

FIG. 6.9. Problem 9: Rarefaction-wall interaction.

TABLE 6.9

Case 9. The rarefaction-wall interaction. Expansion coefficients for output wave strengths (linear model).

\begin{tabular}{|lllll|}
\hline Variable \Coef & Const & \multicolumn{2}{c|}{$w_{1}^{i}$} & \multicolumn{2}{c|}{ Error } \\
& & (r. sonic) & $L_{\infty}$ & STD \\
\hline$w_{1}^{o}$ (l. sonic) & 0.004 & 0.937 & $0.056 \%$ & 0.0001 \\
\hline
\end{tabular}

6.10. Case 10: Rarefaction reflected off of wall passes through contact. The right state velocity $v=0.0$ is fixed, and the left state densities and pressures are held fixed.

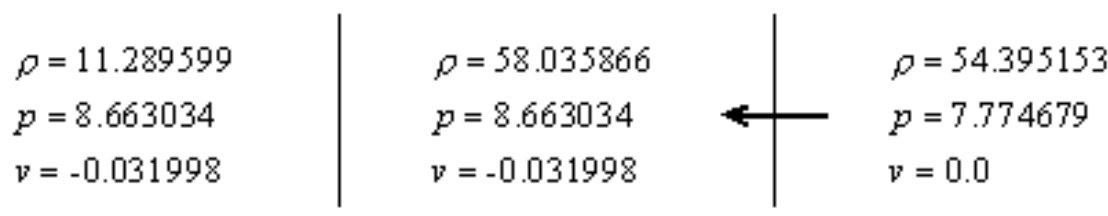

contact (width=8.2) $\quad$ arefaction (width $=7.7$ )

FIG. 6.10. Problem 10: Contact-rarefaction interaction. 
TABLE 6.10

Case 10. The contact-rarefaction interaction (step down). Expansion coefficients for output wave strengths (linear model).

\begin{tabular}{|c|c|c|c|c|c|}
\hline Variable $\backslash$ Coef & Const & $\begin{array}{l}w_{1}^{i} \\
\text { (contact) }\end{array}$ & $\begin{array}{l}w_{2}^{i} \\
\text { (l. sonic) }\end{array}$ & $L_{\infty}$ & $\begin{array}{l}\text { rror } \\
\text { STD }\end{array}$ \\
\hline & \multicolumn{5}{|c|}{$10 \%$ input variation } \\
\hline$w_{1}^{o}$ & 0.007 & 0.013 & 0.641 & $0.45 \%$ & 0.000004 \\
\hline$w_{2}^{o}$ (contact) & 0.453 & 0.327 & 0.008 & $0.16 \%$ & 0.00004 \\
\hline$w_{3}^{o}(\mathrm{r}$. sonic $)$ & -0.009 & 0.013 & 0.930 & $0.57 \%$ & 0.000004 \\
\hline
\end{tabular}

\section{REFERENCES}

[1] I. Babuska And W. Rheinboldt, A posteriori error estimates for the finite element method, Internat. J. Numer. Methods Engrg., 12 (1978), pp. 1597-1615.

[2] L. Bonet-Cunha, D. S. Oliver, R. A. Redner, And A. C. Reynolds, A hybrid Markov chain Monte Carlo method for generating permeability fields conditioned to multiwell pressure data and prior information, Soc. Pet. Eng. J., 3 (1998), pp. 261-271.

[3] A. Chorin And J. Marsden, A Mathematical Introduction to Fluid Mechanics, SpringerVerlag, New York, Heidelberg, Berlin, 1979.

[4] B. CockBurn And H. Gau, A posteriori error estimates of general numerical methods for scalar conservation laws, Mat. Aplic. Comp., 14 (1995), pp. 37-47.

[5] P. S. Craig, M. Goldstein, J. C. Rougier, and A. H. Seheult, Bayesian forecasting for complex systems using computer simulators, J. Amer. Statist. Assoc., 96 (2001), pp. $717-$ 729.

[6] B. DeVolder, J. Glimm, J. W. Grove, Y. Kang, Y. Lee, K. Pao, D. H. Sharp, And K. YE, Uncertainty quantification for multiscale simulations, J. Fluids Engrg., 124 (2002), pp. $29-41$.

[7] J. Glimm, Y.-HA LeE, And K. Ye, A simple model for scale up error, in Fluid Flow and Transport in Porous Media: Mathematical and Numerical Treatment, Contemp. Mathematicians 295, AMS, Providence RI, 2002, pp. 241-251.

[8] J. Glimm, S. Hou, H. Kim, Y. Lee, D. Sharp, K. Ye, and Q. Zou, Risk management for petroleum reservoir production: A simulation-based study of prediction, Comput. Geosci., 5 (2001), pp. 173-197.

[9] J. Glimm, S. Hou, H. Kim, D. Sharp, And K. Ye, A probability model for errors in the numerical solutions of a partial differential equation, CFD Journal, 9 (2000), pp. 485-493.

[10] J. Glimm, S. Hou, Y. Lee, D. Sharp, and K. Ye, Prediction of oil production with confidence intervals, in SPE Reservoir Simulation Symposium, Houston, TX, 2001.

[11] J. Glimm And D. Sharp, An S-matrix theory for classical nonlinear physics, Found. Phys., 16 (1986), pp. 125-141.

[12] J. Glimm AND D. H. Sharp, Stochastic methods for the prediction of complex multiscale phenomena, Quart. Appl. Math., 56 (1998), pp. 741-765.

[13] J. Glimm And D. H. Sharp, Prediction and the quantification of uncertainty, Phys. D, 133 (1999), pp. 152-170.

[14] A. HARTEN, ENO schemes with subcell resolution, J. Comput. Phys., 83 (1989), pp. $148-184$.

[15] B. K. Hegstad AND H. OMRE, Uncertainty assessment in history matching and forecasting, in Geostatistics Wollongong '96, E. Y. Baafi and N. A. Schofield, eds., Kluwer Academic Publishers, Dordrecht, The Netherlands, 1997, pp. 585-596.

[16] Y. KANG, Estimation of Computational Simulation Errors in Gas Dynamics, Ph.D. thesis, State Univ. of New York, Stony Brook, 2003.

[17] M. C. Kennedy And A. O'Hagan, Bayesian calibration of computer models, J. Roy. Statist. Soc. Ser. B, 63 (2001), pp. 425-450.

[18] L. Machiels, A. Patera, J. Peraire, and Y. Maday, A general framework for finite element a posteriori error control: Application to linear and nonlinear convection-dominated problems, in Proceedings of ICFD Conference on Numerical Methods for Fluid Dynamics, Oxford, England, 1998.

[19] J. T. Oden And M. Ainsworth, A Posteriori Error Estimation in Finite Element Analysis, John Wiley and Sons, New York, 2000.

[20] W. Press, S. Teukolsky, W. Vetterling, and B. Flannery, Numerical Recipes in Fortran 77, Cambridge University Press, Cambridge, UK, 1992.

[21] G. B. Whitham, Linear and Nonlinear Waves, John Wiley and Sons, New York, 1974. 